HIAS-E-43

\title{
Exposure Problem in Multi-Unit Auctions
}

\author{
Xin Meng \\ Dongbei University of Finance and Economics \\ Hikmet Gunay \\ Hitotsubashi Institute for Advanced Study, Hitotsubashi University \\ The University of Manitoba
}

February 2017

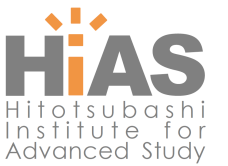

Hitotsubashi Institute for Advanced Study, Hitotsubashi University

2-1, Naka, Kunitachi, Tokyo 186-8601, Japan

tel:+81 425808604 http://hias.ad.hit-u.ac.jp/

HIAS discussion papers can be downloaded without charge from:

http://hdl.handle.net/10086/27202

https://ideas.repec.org/s/hit/hiasdp.html

All rights reserved. 


\title{
EXPOSURE PROBLEM IN MULTI-UNIT AUCTIONS
}

\author{
Xin Meng \\ Dongbei University of Finance and Economics \\ and \\ Hikmet Gunay* \\ Hitotsubashi Institute for Advanced Study, Hitotsubashi University \\ and the University of Manitoba
}

February 10, 2017

\begin{abstract}
We characterize the optimal bidding strategies of local and global bidders for two heterogeneous licenses in a multi-unit simultaneous ascending auction (SAA) like the one used in the 2008 Canadian Advanced Wireless Spectrum license auction. The global bidder wants to win both licenses to enjoy synergies; therefore, she bids more than her stand-alone valuation of a license. This exposes her to the risk of losing money even when she wins all licenses. We determine the optimal bidding strategies in the presence of an exposure problem. By using simulation methods, first, we show that the probability of inefficient allocations in the simultaneous ascending auction can be up to 9 per cent. Second, we show that the global bidder can end up with a loss with 6 per cent probability depending on the distribution. We also investigate the relation between inefficient allocation and the revenue of SAA and VCG auctions.
\end{abstract}

JEL Codes: D44, D82

Keywords: Multi-Unit Auctions, Vickrey Clarke Groves (VCG) mechanism, Exposure Problem, Synergies, Complementarity, Spectrum License Auction

\footnotetext{
*Authors contributed equally to this paper. Xin Meng acknowledges the support of NSFC(National Natural Science Foundation of China under Grant No.71302180), SSHRC and the support of DUFE-CIBO(Subject Research Project Fund under Grant No.LNTX2015001). H. Gunay thanks Osaka University-ISER for hosting him while doing this research, SSHRC for an indirect support, and the University of Manitoba for providing internal grants. We thank M. Aoyagi, D. Mishra, P. Murto, M. Pak, L. Ulku, J. Valimaki, R. Wang, and anonymous reviewers for their comments on the (earlier versions of the) paper. The usual disclaimer applies. This paper has been presented at Aalto University, Florida State University, Hitotsubashi University, ISI-Delhi, IIM-Bangalore, METU, Kyoto University-KIER,Osaka University-ISER, SWUFE-RIEM, Shanghai Jiao Tong University, TOBB-ETU, 2009 Iowa Alumni Workshop, 5th CIREQ PhD Students' Conference, 43rd CEA meetings held in Toronto, 2009 Midwest Economic Theory Conference held at Penn State University, ESAM 2011, and 2014 AMES held in Academia Sinica. We thank the participants for their feedback.
} 


\section{Introduction}

In the recent Canadian Advanced Wireless Spectrum (AWS) license auction, hundreds of (heterogenous) licenses were sold to firms simultaneously. Each of these licences gave the spectrum usage right of a geographical area to the winning firm. Rogers spent almost $\$$ CAD 1 billion to buy spectrum rights in each province and territory. However, Manitoba Telecom Services only bought licenses for Manitoba, and SaskTel only bought licenses for Saskatchewan. ${ }^{1}$ Firms such as Manitoba Telecom Services, and SaskTel are called 'local' firms since they are interested in winning only specific licenses in order to serve local markets while firms such as Rogers are called 'global' firms since they are interested in winning licenses across the nation. ${ }^{2}$ The global firms enjoy synergies if they win all the licenses; this gives them an incentive to bid more than their stand-alone valuations for some licenses. As a result, this bidding has the risk of incurring losses. Therefore, global bidders may lower their bids. This is known as the exposure problem.

In a model simplifying the recent Canadian Advanced Wireless Spectrum license auctions, ${ }^{3}$ we derive the optimal bidding strategies of local and global firms in a simultaneous ascending auction (SAA) of two "heterogeneous" licenses when there is the possibility of ex-post loss. Although one of the main concerns of the policy makers is the efficiency of the spectrum auction (Cramton and Schwartz, 2000), we are not aware of any paper in the literature that calculates the probability of the auction resulting in an inefficient outcome. We show that this probability is up to 9 per cent for some distributions in our main model.

In order to study the effect of the inefficient outcomes on revenue, we divide the inefficient allocations into two groups. In the first group, the global bidder wins one license or all licenses with an (ex-post) loss. We show that the SAA auction has a higher revenue than the efficient VCG auction for these kind of inefficient outcomes (ex-post); the seller has a higher revenue

\footnotetext{
${ }^{1}$ Information on Canadian AWS auction is mainly taken from Industry Canada Website.

${ }^{2}$ Firms must deposit money before the auction. The amount of money shows their intention whether they will bid for all licenses or only select ones. Also, given the firms previous serving areas, one can expect whether a firm is local or global.

${ }^{3}$ Some recent US license auctions also follow a similar format.
} 
at the expense of the global bidder. Our simulations showed that the global bidder might end up with a loss 6 per cent of the time for some distribution functions. In the second group, the global bidder does not bid high enough from the efficiency point of view, and loses all licenses or wins one license with a profit (where as the efficient outcome requires the global bidder to win both). Since the VCG auction corrects this inefficiency problem, it results in a higher revenue than the SAA auction (ex-post). We believe that this is a support for the existence of exposure problem since the global bidder does not bid high enough.

The current literature on multi-unit auctions generally assumes that global bidders have either equal valuations (Englmaier et. al (2009), Kagel and Levin (2005), Katok and Roth (2004), Albano et. al. (2001), Rosenthal and Wang (1996), and Krishna and Rosenthal (1996)), very large synergies (Albano et al. (2006)), or that the marginal value of an additional license depends only on the number of licenses already held (Goerre and Lien (2014)). The spectrum licenses for different geographic areas are not homogenous objects; hence, the equal valuation assumption or equal marginal valuation of licenses assumption does not fit the Canadian AWS (and US) spectrum license auction. Specifically, we model situations in which one spectrum license for, say, Toronto is more valuable than the spectrum license for Manitoba. ${ }^{4}$ We do this by drawing two valuations from a distribution function and assign the maximum value for one license (say for Toronto license) and the minimum value for the other license. This modelling assumption ensures that the auction for the weak location concludes before the strong location. This simplifies finding the equilibrium. Since there may be other auctions that this assumption might not hold, we also study a revised model where the valuations of the local bidders are independently drawn. In the revised model, there is a positive probability that auctions in any location might end first. Our main contribution to the literature is the result of our simulations, and characterizing the equilibrium for heterogenous licenses when synergies are moderate. By using the equilibrium and simulations, we calculate the probability of inefficient allocations, expected average revenue for SAA and VCG auctions, and expected welfare loss from the SAA auction by using ex-post

\footnotetext{
${ }^{4}$ The winning bids on the Industry Canada website show that Toronto licenses are more valuable than the Manitoba licenses.
} 
valuations, and show the link between inefficient allocations and revenue.

\section{The Model}

Suppose there are 2 licenses for sale: license $A$ and $B .^{5}$ There is one global bidder who demands both licenses and $m$ local bidders who demand only license $A$, and another different $m$ local bidders who demand only license $B$. To determine the valuations of global bidder, we draw two private valuations, $X_{0}$ and $Y_{0}$ from the commonly known distribution function $F($.$) . The global bidder's stand alone valuation for license A$ is $v_{0 A}=\operatorname{Max}\left\{X_{0}, Y_{0}\right\}$, and his stand alone valuation for license $B$ is $v_{0 B}=\operatorname{Min}\left\{X_{0}, Y_{0}\right\}$. We want to model heterogeneous licenses, so with this modeling we make sure that license $A$ is more valuable (since it is a license for a big city like Toronto) than license $B$ (since it is a license for a medium size city like Winnipeg) for the global bidder. The global bidder's total valuation, given that it wins two licenses is, $V_{0}=v_{0 A}+v_{0 B}+\theta$, where the synergy term $\theta>0$ is assumed to be private knowledge, and it has a continuous density function on $[0, a)$ where $a \in(0, \infty]{ }^{6}$ To determine the valuations for local bidders, we draw two private valuations $X_{i}$ and $Y_{i}$ for $i=1,2, \ldots, m$, from the same distribution function $F($.$) . The valuation of local bidder i A$ for license $A$ is $v_{i A}=\operatorname{Max}\left\{X_{i}, Y_{i}\right\}$, and the valuation of local bidder $i B$ for license $B$ is $v_{i B}=\operatorname{Min}\left\{X_{i}, Y_{i}\right\}$. The distribution function $F($.$) has a support on [0,1]$ and probability density function $f($.$) which is positive everywhere with the only exception that f(0) \geq 0$ is allowed. The bidders' type, global or local, is publicly known. The valuations of global and local bidders are private information. These valuations result in $\operatorname{Max}\{v i A: i=1,2, \ldots, m\} \geq$ $\operatorname{Max}\{v i B: i=1,2, \ldots, m\} .^{7}$ Later, we add a model where the local bidders' valuations are drawn independently, and hence, the license $B$ might be more valuable than license $A$.

\footnotetext{
${ }^{5}$ We use two licenses like Albano et. al. (2001 and 2006), Brusco and Lopomo (2002), and Menucicci (2003).

${ }^{6}$ It will be clear later that the distribution of $\theta$ does not matter as long as we have one global bidder.

${ }^{7}$ With this formulation, it is possible that a few local bidders $B$ may have a high valuation than some other local bidder $A$ due to them being very cost effective. Also, note that we may assume different number of local bidders on each license and qualitative results will not change, as long as the number of local bidders $A$ is higher than the local bidders $B$. This will guarantee that max value for license $A$ is greater than max value for license $B$. We relax this assumption later.
} 
We consider a setting where the licenses are auctioned off simultaneously through an ascending multi-unit auction. Each license is auctioned off at a different auction (like Krishna and Rosenthal (1996) but unlike Kagel and Levin (2005)) at the same time. Prices start from zero for both licenses and increase simultaneously and continuously at the same rate. Bidders choose when to drop out. When only one bidder is left on a given license, the clock stops for that license, and the sole remaining bidder wins the license at the price at which the last bidder drops out. If there is more than one bidder remaining on the other license, its price will continue to increase. If $m$ bidders drop out at the same price and nobody is left in the auction, then each one of them will win the license with probability $\frac{1}{m}$. This is a zero measure event given the valuations are drawn from a continuous distribution function.

The drop-out decision is irreversible. Once a bidder drops out of bidding for a given license, he cannot bid for this license at a later time. ${ }^{8}$ The number of active bidders and the drop-out prices are publicly known. We also assume that there is no budget constraints for the bidders.

Local bidders have a weakly dominant strategy to stay in the auction until the price reaches their valuation. We assume all bidders will use their weakly dominant strategy in equilibrium. We derive a symmetric Bayesian-Nash equilibrium in what follows.

To understand the global bidder's strategy, consider a subgame in which every local bidder drops out of license $B$ auction, and hence, the global bidder wins license $B$ at the price $p_{B}=\max \left\{v_{1 B}, \ldots, v_{m B}\right\}$ since local bidders use their weakly dominant strategy and bid up to their valuation. The global bidder knows that, at a given clock price $p_{c}<1$, it will win license $A$ at the price $p_{A}=\max \left\{v_{1 A}, \ldots, v_{m A}\right\}$. The distribution of each $v_{i A}$ is $\left[F\left(v_{i A} \mid p_{c}\right)\right]^{2}$ given the clock price $p_{c} \cdot{ }^{9}$ Then the conditional distribution of $p_{A}$ is the highest order statistic which we will denote as $G$ where $G\left(p_{A} \mid p_{c}, m_{A}\right)=\left[F\left(p_{A} \mid p_{c}, m_{A}\right)\right]^{2 m_{A}}=\left(\frac{\int_{p_{c}}^{p_{A}} f_{A}(v) d v}{\int_{p_{c}}^{1} f_{A}(v) d v}\right)^{2 m_{A}}$, where

\footnotetext{
${ }^{8}$ In the real-world auctions, there is an activity rule: if the bidders do not have enough highest standing bids, then the number of licenses they may bid on is decreased (in the next rounds). Hence, when there are two licenses, this translates into an irreversible drop-out.

${ }^{9}$ If the local bidder's valuations were independent, the conditional distribution of $v_{i A}$ given the clock price $p_{c}$ would be $F\left(v_{i A} \mid p_{c}\right)$. Since the valuations comes from a max distribution, each $v_{i A}$ is distributed as $\left[F\left(v_{i A} \mid p_{c}\right)\right]^{2}$.
} 
$m_{A}$ is the number of active local $A$ bidders. Then to determine its optimal drop out price $p_{A}^{*}$ for license $A$, the global bidder will maximize its continuation payoff. ${ }^{10}$ The payoff is

$$
\max _{p_{A}^{*}} v_{0 B}-p_{B}+\int_{p_{c}}^{p_{A}^{*}}\left(v_{0 A}+\theta-p_{A}\right) d G\left(p_{A} \mid p_{c}, m_{A}\right)
$$

The first order condition is

$$
\left(v_{0 A}+\theta-p_{A}^{*}\right) d G\left(p_{A} \mid p_{c}, m_{A}\right)=0 \quad \Rightarrow \quad p_{A}^{*}=v_{0 A}+\theta
$$

It is easy to check the second order condition which shows that this is a unique maximizer. ${ }^{11}$ The result is intuitive, the global bidder bids until the price reaches its marginal value of license $A$. The number of active local bidders does not affect the decision of the global bidder. We would also like to note that the optimal drop out price can be found by comparing payoffs from two cases. CASE 1 is the payoff from dropping out of license $A$ auction at the clock price $p_{c}$ (without winning license A), and this payoff is $v_{0 B}-p_{B}$. CASE 2 is the payoff from continuing on license $\mathrm{A}$ auction and winning at price $p_{c}$ (with probability 1). This payoff is $v_{0 A}+v_{0 B}+\theta-p_{B}-p_{c}$ which is monotonically decreasing in the price $p_{c}$. Clearly as long as the payoff from CASE 2 is higher than the payoff from CASE 1, the global bidder will continue to stay in license A auction. The updated optimal drop-out price, $p_{A}^{*}$, is found by equating these two equations:

$$
v_{0 A}+v_{0 B}+\theta-p_{B}-p_{A}^{*}=v_{0 B}-p_{B} \Rightarrow p_{A}^{*}=v_{0 A}+\theta
$$

Similarly, if the global bidder wins license $A$, then it stays in license $B$ auction until the price reaches $v_{0 B}+\theta$, but this cannot happen in equilibrium since $p_{A}>p_{B}$ and local bidders use weakly dominant strategy. ${ }^{12}$

\footnotetext{
${ }^{10}$ With a slight abuse of notation, we treat $p_{A}^{*}$ as a variable and the point of optimal drop out price.

${ }^{11}$ The second order condition is $\left[\left(v_{0 A}+\theta-p_{A}\right) d G\left(p_{A} \mid p_{c}, m_{A}\right)\right]-d G\left(p_{A} \mid p_{c}, m_{A}\right)$ but the first term in closed brackets is zero at $p_{A}=p_{A}^{*}$ from the first order condition, and $-d G\left(p_{A} \mid p_{c}, m_{A}\right)<0$ for all $p_{c}<1$. The function is strictly concave at its unique critical point.

${ }^{12}$ In the revised model, this can be an equilibrium.
} 
If the global bidder's average valuation, $\frac{v_{0 A}+v_{0 B}+\theta}{2}$, exceeds 1 , its equilibrium strategy is bidding anything from 1 up to his average valuation. As a result, the global bidder outbids his rivals. If $\theta$ is large enough, this condition is always satisfied.

To calculate the optimal drop-out price for the global bidder for license $B$ when his average valuation is less than 1 , one should maximize the global bidder's expected payoff. The expected payoff for the global bidder, in which $p$ denotes the drop out price for license $B$, is:

$$
\begin{array}{r}
\max _{p}\left[\int _ { p _ { c } } ^ { p } \left[\int_{p_{B}}^{\min \left\{v_{0 A}+\theta, 1\right\}}\left(v_{0 A}+\theta-p_{A}+v_{0 B}-p_{B}\right) d G\left(p_{A} \mid p_{B}, m_{A}\right)\right.\right. \\
\left.\quad+\int_{\min \left\{v_{0 A}+\theta, 1\right\}}^{1}\left(v_{0 B}-p_{B}\right) d G\left(p_{A} \mid p_{B}, m_{A}\right)\right] d H\left(p_{B}\right) \\
\left.+\int_{p}^{\max \left\{p, v_{0 A}\right\}}\left[\int_{p_{B}}^{v_{0 A}}\left(v_{0 A}-p_{A}\right) d G\left(p_{A} \mid p_{B}, m_{A}\right)\right] d H\left(p_{B}\right)\right]
\end{array}
$$

In this equation $H\left(p_{B}\right)=\left[1-\left(1-F(.)^{2}\right]^{m_{B}}\right.$ since each valuation of local bidder $\mathrm{B}$ has a cdf of $\left[1-\left(1-F(.)^{2}\right)\right]$, and the maximum of these when there are $m_{B}$ local bidders $B$ is $H\left(p_{B}\right){ }^{13}$ Readers will see that, in the proofs, $H\left(p_{B}\right)$ has no direct role in calculating the optimal drop out price but it has a role in calculating the second order condition. We explain this in footnote 16 .

The explanation for the expected payoff equation, given the sequential rationality of the global bidder, is as follows. The first line calculates the expected payoff for the global bidder from winning both license $B$ and (then) license $A$. The outer integral has an upper limit $p$ since $p_{B}$ must be distributed between $p_{c}$ and $p$ for the global bidder to win license $B$. After winning license $B$, global bidder will stay in license $A$ auction until $v_{0 A}+\theta$ by the sequential rationality (or will win license $A$ as long as it stays until the price reaches 1.). The second line shows the expected payoff from winning license $B$ but losing $A$. In order to lose license $A$ after winning license $B$, the condition $p_{A}>v_{0 A}+\theta$ must hold given the

\footnotetext{
${ }^{13}$ Recall that each local bidder B's valuation is a minimum random variable; hence, the cdf of minimum of two independently drawn random variables from $F$ is $\left[1-\left(1-F(.)^{2}\right)\right]$.
} 
sequential rationality of the global bidder. The third line shows the expected payoff from winning license $A$ only. For this to happen, $p_{B}>p$ must hold (to lose license $B$ ) and then since $p<p_{B}<p_{A}$, we have the lower limit of the integral as $p_{B}$. The upper limit of the integral shows the sequential rationality of the global bidder. The global bidder who loses license $B$ becomes just like a local bidder and will bid until $v_{0 A}$.

When $v_{0 A}+\theta<1$, the first derivative of this maximization problem is:

$$
\begin{array}{r}
F O C=\left[\int_{p}^{v_{0 A}+\theta}\left(v_{0 A}+v_{0 B}+\theta-p-p_{A}\right) g\left(p_{A} \mid p, m_{A}\right) d p_{A}+\int_{v_{0 A}+\theta}^{1}\left(v_{0 B}-p\right) g\left(p_{A} \mid p, m_{A}\right) d p_{A}\right. \\
\left.-\mathbb{1}_{\left[p<v_{0 A}\right]} \int_{p}^{v_{0 A}}\left(v_{0 A}-p_{A}\right) g\left(p_{A} \mid p, m_{A}\right) d p_{A}\right] h(p)
\end{array}
$$

We can write this as $F O C=J\left(p, m_{A}\right) h(p)$, where $J($.$) is the term in the closed brackets.$ First, note that the FOC is continuous at $p=v_{0 A} \cdot{ }^{14}$ Second, any $p_{0}^{*}$ that solves $J\left(p, m_{A}\right)=0$ also solves $F O C=0$. In the proofs, we will show that, this $p_{0}^{*}$ is indeed the unique solution to the FOC problem. Third, note that when FOC $\left(p_{0}^{*}\right)=0, h(p)$ is cancelled out from FOC. Let us discuss the first order condition of this maximization problem after cancelling $h(p)$. The global bidder must compare the payoffs for two cases at each potential drop out price $p$ as the clock is running: Case $\mathbf{1}$ is the payoff from dropping out from license B auction at price $p$ (without winning) and optimally continuing to bid on the license A auction (or optimally drop out from license A auction at the same $p$, if $p>v_{0 A}$ ). The payoff is:

$$
E \Pi_{0}^{1}\left(p, m_{A}\right)=\mathbb{1}_{\left[p<v_{0 A}\right]} \int_{p}^{v_{0 A}}\left(v_{0 A}-p_{A}\right) g\left(p_{A} \mid p, m_{A}\right) d p_{A}
$$

This is equation 6 above when $v_{0 A}+\theta<1$ after cancelling $h(p)$. Case 2 is the payoff from winning license $\mathrm{B}$ at price $\mathrm{p}$ (with probability 1 so this is the highest price the global bidder would pay) and optimally continuing on the license A auction.

$$
\begin{aligned}
E \Pi_{0}^{2}\left(p, m_{A}\right)=\int_{p}^{\operatorname{Min}\left\{v_{0 A}+\theta, 1\right\}} & \left(v_{0 A}+v_{0 B}+\theta-p-p_{A}\right) g\left(p_{A} \mid p, m_{A}\right) d p_{A} \\
& +\int_{M i n\left\{v_{0 A}+\theta, 1\right\}}^{1}\left(v_{0 B}-p\right) g\left(p_{A} \mid p, m_{A}\right) d p_{A}
\end{aligned}
$$

\footnotetext{
${ }^{14}$ As $p$ approaches $v_{0 A}$ from the right, the value of Equation 6 is always zero. As it approaches from the left, the value of Equation 6 approaches to zero.
} 
This is equation 5 above (when $v_{0 A}+\theta<1$ ) after cancelling $h(p)$. Hence, as long as the payoff of Case 2 is higher, the global bidder will stay in license $B$ auction. In the proofs, we show that $J\left(p, m_{A}\right)$ is decreasing in $p$. Then, we will show that $J\left(p=v_{0 B}, m_{A}\right)>0$ and $J\left(p=v_{0 A}+\theta, m_{A}\right)<0$. Hence, if $J\left(v_{0 A}, m_{A}\right)<0$, there is a unique root (solution) $p_{0}^{*} \in\left(v_{0 B}, v_{0 A}\right)$. If not, we will have a unique root (solution) $p_{0}^{*} \in\left[v_{0 A}, \min \left\{v_{0 A}+\theta, 1\right\}\right)$. We show that this is the optimal drop-out price, $p_{0}^{*}$, which is the solution to the first order condition of the global bidder's expected payoff maximization problem. ${ }^{15}$

In Proposition 1 below, we characterize the global bidder's equilibrium bids. Note that these payoffs are changing as local bidders bidding for $A$ are dropping out; that is, $m_{A}$ is changing. Therefore, the lemma below gives the global bidder's (updated) equilibrium dropout price as the local bidders drop out. In the proof of Proposition 1, we show that this updated price increases as local bidders drop out of license $A$ auction. ${ }^{16}$

Proposition 1 Suppose that the average valuation of the global bidder is less than 1 and there are $m_{A}$ local bidders bidding on license $A$ where $m_{A} \geq 1$ and there is at least one active local bidder $B$.

The global bidder will maximize its payoff by dropping out of the license B auction at the unique optimal drop-out price $p_{0}^{*}\left(m_{A}\right)$ that satisfies $J\left(p, m_{A}\right)=0$. Moreover,

a) If $v_{0 A}+\theta<1$, and $J\left(v_{0 A}, m_{A}\right)=\int_{v_{0 A}}^{v_{0 A}+\theta} G\left(p_{A} \mid v_{0 A}, m_{A}\right) d p_{A}+\left(v_{0 B}-v_{0 A}\right)<0$, then $p_{0}^{*}\left(m_{A}\right)<v_{0 A}$ and the global bidder will stay in the license $A$ auction until $v_{0 A}$ (after dropping out from the license $B$ auction).

b) If $v_{0 A}+\theta<1$, and $J\left(v_{0 A}, m_{A}\right)=\int_{v_{0 A}}^{v_{0 A}+\theta} G\left(p_{A} \mid v_{0 A}, m_{A}\right) d p_{A}+\left(v_{0 B}-v_{0 A}\right) \geq 0$, then $p_{0}^{*}\left(m_{A}\right) \geq v_{0 A}$ and the global bidder will also drop out of the license $A$ auction at $p_{0}^{*}\left(m_{A}\right)$.

c) If $v_{0 A}+\theta \geq 1$, and $J\left(v_{0 A}, m_{A}\right)=\int_{v_{0 A}}^{1} G\left(p_{A} \mid v_{0 A}, m_{A}\right) d p_{A}+\left(v_{0 B}+\theta-1\right)<0$, then $p_{0}^{*}\left(m_{A}\right)<v_{0 A}$ and the global bidder will stay in the license $A$ auction until $v_{0 A}$ (after dropping

\footnotetext{
${ }^{15}$ The comparing payoffs method is used by Albano et. al. (2001) for identical licenses.

${ }^{16}$ Note that when $v_{0 B}<v_{0 A}$, local bidders $\mathrm{B}$ dropping out of auction do not affect the global bidder's drop out price. This seemingly surprising result arises because the global bidder's equilibrium incentives are conditioned on obtaining license $\mathrm{B}$ at current price $p$ so that the remaining number of local bidders $\mathrm{B}$ is irrelevant. The number of local bidders $\mathrm{A}$ is important since it affects the price at which the global bidder can obtain that license. We are grateful to a referee for making this point.
} 
out from the license $B$ auction). ${ }^{17}$

d) If $v_{0 A}+\theta \geq 1$, and $J\left(v_{0 A}, m_{A}\right)=\int_{v_{0 A}}^{1} G\left(p_{A} \mid v_{0 A}, m_{A}\right) d p_{A}+\left(v_{0 B}+\theta-1\right) \geq 0$, then $p_{0}^{*}\left(m_{A}\right) \geq v_{0 A}$ and the global bidder will also drop out of the license $A$ auction at $p_{0}^{*}\left(m_{A}\right)$.

e) As $m_{A}$ decreases, $p_{0}^{*}$ increases.

Proof. See Appendix.

The inequality conditions on $\int_{v_{0 A}}^{1} G\left(p_{A} \mid v_{0 A}, m_{A}\right) d p_{A}+\left(v_{0 B}+\theta-1\right)$ in part c and d just make the first order condition at $p=v_{0 A}$ less than or greater than zero for the $v_{0 A}+\theta>1$ case. We also note that $J\left(p, m_{A}\right)=0$ is equivalent to $E \Pi_{0}^{1}\left(p, m_{A}\right)=E \Pi_{0}^{2}\left(p, m_{A}\right)$ for all cases from part a to d.

We are ready to summarize our Bayesian-Nash equilibrium.

\section{Proposition 2 (Bayesian-Nash Equilibrium)}

a) A local bidder of each license will stay in the auction $j$ until the price reaches her valuation $v_{i j}$ where $j=\{A, B\}, i=\{1,2,3, . . m\}$.

b) A global bidder active only on license $j$ will bid $v_{0 j}+\theta$, if he won the other license. He will bid $v_{0 j}$ if he dropped out of the other license.

c) When the average valuation is less than one, the global bidder who is active on both licenses and facing $m_{A}$ active local bidders on license $A$ will behave as described in proposition 1.

d) If the average valuation is greater than one, the global bidder will stay in both auctions until price reaches his average valuation.

\section{Simulations; Inefficient Allocations And The Rev- enue Comparison of SAA and VCG Auctions}

Policy makers want an efficient auction outcome (see Cramton and Schwartz, 2000). If the outcome is inefficient, the winning firms may choose not to use the scarce spectrum after

\footnotetext{
${ }^{17}$ While $J\left(p, m_{A}\right)$ takes different forms for different cases depending on $v_{0 A}+\theta<1$ or not, we keep using the same notation since it is clear which case we are referring to.
} 
the auction, and hence, the society cannot benefit from the auction fully. ${ }^{18}$ Despite this clear policy objective, the probability of inefficient SAA outcome has not been calculated in the literature, to our best knowledge. We believe that the reason was that an analytic calculation is not possible, and one has to use simulations for this. ${ }^{19}$

Our simulation code is written in MATLAB. It starts with drawing two independent valuations for the global firm from the same distribution function. ${ }^{20}$ The global bidder's valuations for license $A$ and $B$ become the maximum and the minimum of these two valuations, respectively. Then, we draw two independent valuations from the same distribution function. We use one local bidder for each license. The local bidder $A$ 's and $B$ 's valuations take the maximum and the minimum of these valuations, respectively. One set of valuations corresponds to one auction. Knowing these valuations, by using our theoretical model, we calculate the optimal drop-out price(s) for the global bidder for all possible different cases. We count how many times, ex-post, the outcome will be inefficient, and what the revenue would be for four different synergy values of $0.2,0.4,0.6,0.8$. Since our results converge when we run for 10000 auctions, we divide the number of inefficient outcomes with 10000 , and derive the probability of an inefficient outcome.

\subsection{Inefficient Outcome}

In order to classify an outcome as efficient or inefficient, we use the following lemma and discussions which are summarized in Table 1.

Lemma 3 If the global bidder has an (ex-post) loss, then $p_{0}^{*}>p_{B}>v_{0 B}$ must hold and the outcome is always inefficient.

This lemma shows that a necessary condition for the global bidder to make a loss is that it must win license $B$ with an (initial) loss. This initial loss may turn into a profit if the

\footnotetext{
${ }^{18}$ One can give an example of inefficient allocation of AWS auction. Quebecor won $10 \mathrm{Mhz}$ of spectrum for Toronto area but had not used it until 2012. According to The Globe and Mail article published on September 19, 2012, "Mr. Pruneau, chief financial officer of Quebecor, confirmed Wednesday that Quebecor has no plans to build a mobile network in Toronto with that $10 \mathrm{MHz}$ of spectrum."

${ }^{19}$ Calculating ex-ante probability might only be possible with numerical methods but this will be more difficult than the method of using ex-post valuations that we use in this paper.

${ }^{20}$ This code is more than 300 pages long as a word document. Codes can be requested from the authors.
} 
global bidder wins license $A$ but it may also end up in an ex-post loss. The latter will be an inefficient outcome. The (ex-post) revenue with SAA auction will be higher for such cases as we will prove later.

This lemma does not give a sufficient condition. We have cases where the global bidder makes a profit but the outcome may be efficient or inefficient (line 3 and 4 of Table 1). Also, note that a case where the global bidder wins only license A and makes a loss is not possible (line 5 of Table 1 ). The reason is that when global bidder loses license $B$, it behaves just like a local bidder and will bid up to $v_{0 A}$.

We summarize all possible outcomes in Table 1.

\begin{tabular}{cccccc} 
& License A won by & License B won by & Global bidder makes & Allocation is & Revenue SAA \\
\hline 1. & Global Bidder & Global Bidder & Profit & Efficient & $p_{A}+p_{B}$ \\
2. & Global Bidder & Global Bidder & Loss & Inefficient & $p_{A}+p_{B}$ \\
3. & Global Bidder & Local Bidder B & Profit & Efficient & $p_{A}+p_{0}^{*}$ \\
4. & Global Bidder & Local Bidder B & Profit & Inefficient & $p_{A}+p_{0}^{*}$ \\
5. & Global Bidder & Local Bidder B & Loss (not possible) & N/A & N/A \\
6. & Local Bidder A & Global Bidder & Loss & Inefficient & $v_{0 A}+\theta+p_{B}$ \\
7. & Local Bidder A & Global Bidder & Profit & Efficient & $v_{0 A}+\theta+p_{B}$ \\
8. & Local Bidder A & Local Bidder B & Zero Profit & Efficient & $p_{0}^{*}+v_{0 A}$ \\
9. & Local Bidder A & Local Bidder B & Zero Profit & Inefficient & $p_{0}^{*}+v_{0 A}$ \\
10. & Local Bidder A & Local Bidder B & Zero Profit & Efficient & $2 p_{0}^{*}$ \\
11. & Local Bidder A & Local Bidder B & Zero Profit & Inefficient & $2 p_{0}^{*}$
\end{tabular}

Table 1: All efficient and inefficient outcomes.

As written in Table 1, there are cases in which the local bidder may win both licenses and the outcome may be efficient or inefficient. For such inefficient cases, the global bidder may drop from both licenses at $p_{0}^{*}$ (line 10 and 11) or may drop from license $B$ at $p_{0}^{*}$ and from license $A$ at $v_{0 A}$ (line 8 and 9). The global bidder does not bid high enough due to the risk of ex-post loss. As we will show later, VCG mechanism corrects this inefficiency, and also increases the revenue (ex-post). These cases are the basis of the exposure problem.

By using Table 1, we can count the number of efficient and inefficient outcomes in our simulations for each of our 10000 auctions. Our simulation results are summarized in Figure 1 and 2. The Ex-post Loss Probability is the summation of cases/auctions that fall under row 2 and 6 in Table 1. Probability of Inefficiency is the summation of cases/auctions that 
Figure 1: Beta Distribution $\alpha=1, \beta=3$.
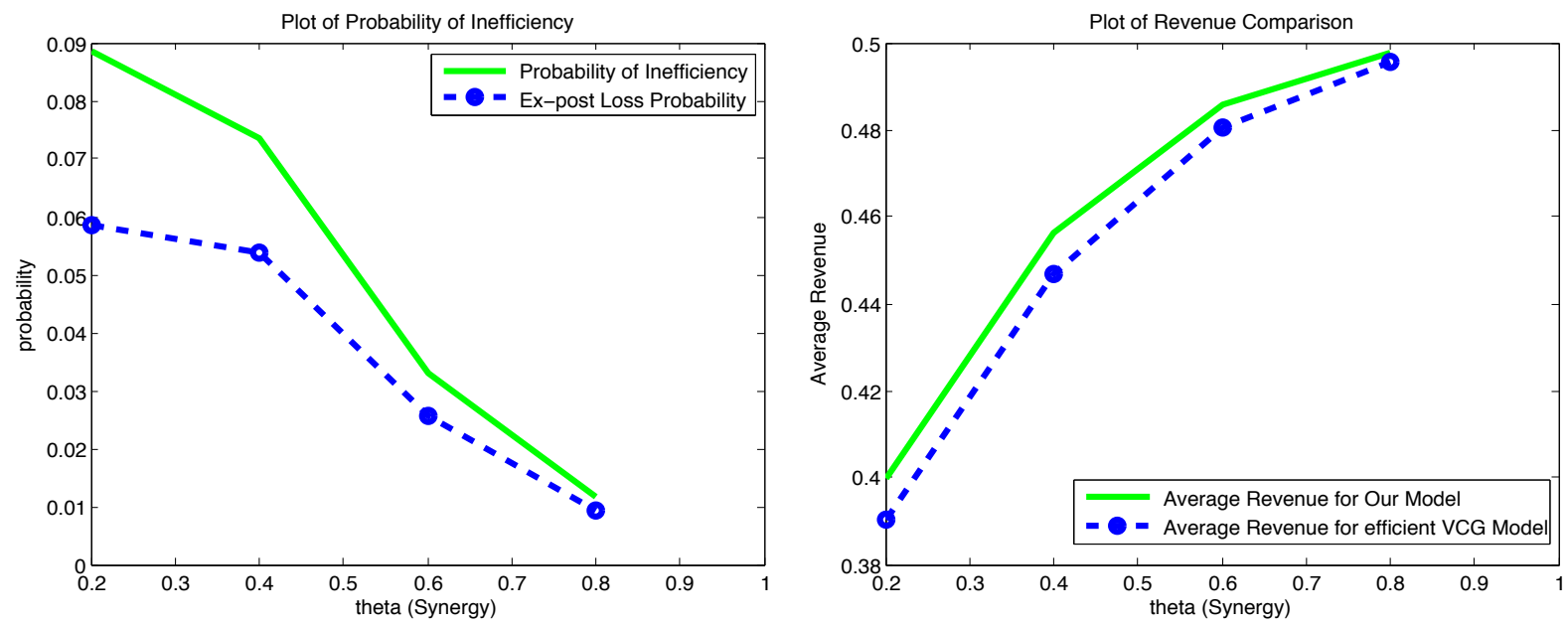
Figure 2: Top: Uniform Distribution. Bottom: Beta Distribution with $\alpha=5, \beta=5$
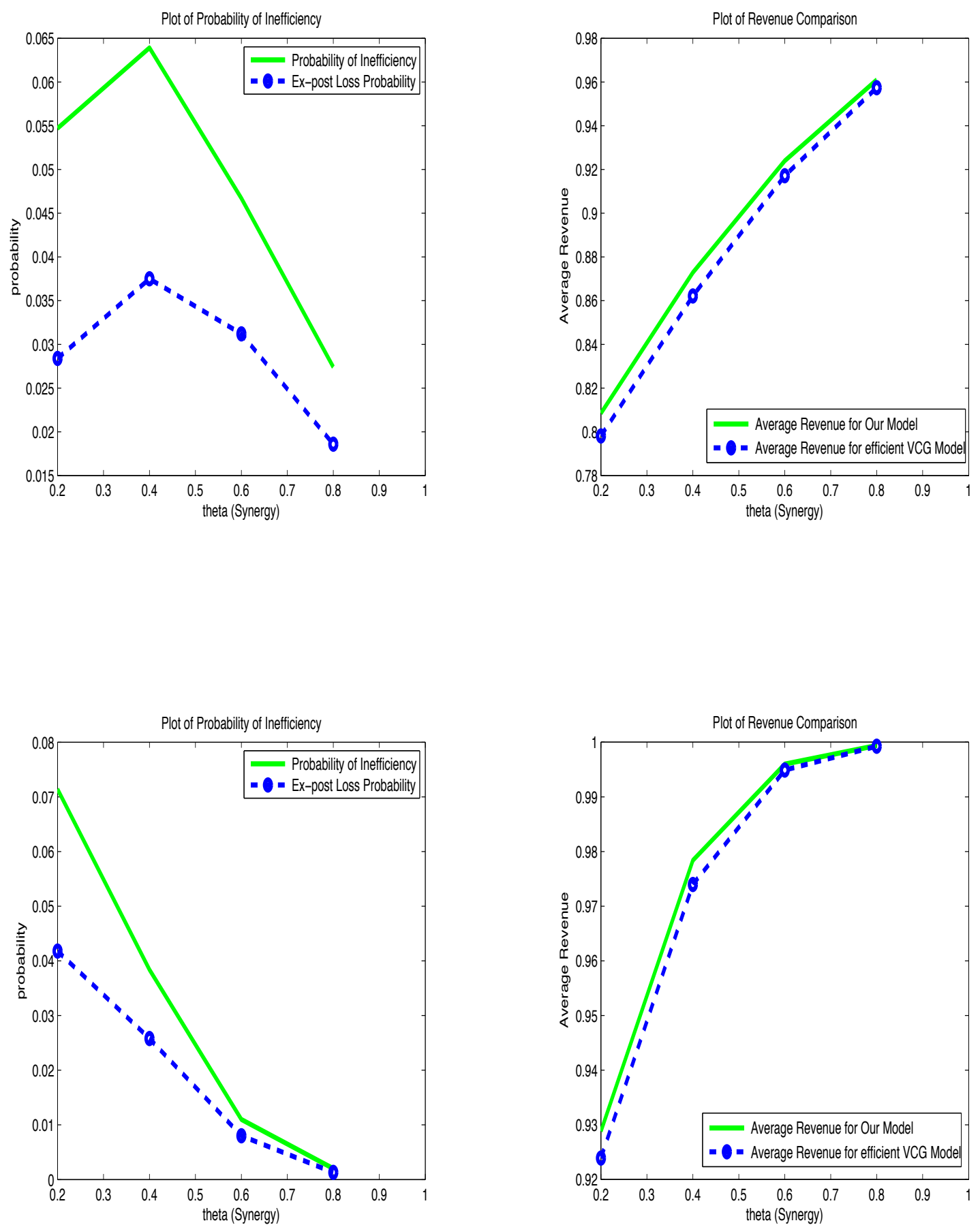
fall under row 2, 4, 6, 9, and 11 .

When the synergy level is 0 (no externality) or when it is greater than 2 , the outcome is always efficient with the SAA auction. However, for in between values, there are inefficient allocations according to the figures; hence, there is not a monotonic relation between synergy levels and the probability of inefficient allocations. This is what we observe in the uniform distribution figure. ${ }^{21}$

Our main result is that the inefficient outcomes can be as high as 9 per cent. The cases where the global bidder makes a loss accounts for approximately 6 per cent of the total outcome in some simulations (see Figure 1). There are cases where local bidders winning inefficiently. This implies that the global bidder is not bidding as high as it should from an efficiency point of view since it is well aware of the possibility of making a loss.

In Table 2, we report the optimal drop out prices for a sample of global bidder's valuations. The table shows that the global bidder exposes herself to more risk by bidding well-over her stand alone valuation of license $B$ when her synergy level is higher and/or when she expects local bidder A's valuation to be lower. However, as the synergy increases by 0.2 unit, the optimal drop out price increases less than 0.2 unit.

The implication of these on the revenue of the SAA and the VCG auction is an interesting one. When the local bidders win one or both licenses inefficiently, the revenue of the VCG auction is always greater than the revenue of the SAA auction ex-post. When the global bidder wins licenses inefficiently (with an ex-post loss), the revenue of SAA auction is always greater than the revenue of the VCG auction ex-post. This is not surprising since the global bidder bids over its stand alone valuation, it ends up with an ex-post loss but this increases the seller's revenue.

We summarize the discussion above with the following propositions which explain the impact of inefficient allocations on revenue.

Proposition 4 If the global bidder wins licenses with an ex-post loss then the revenue of the

\footnotetext{
${ }^{21}$ In the other figures, the peak of the graph must be below 0.2 . The figures (x-axis) start from 0.2 and hence show only a decreasing part.
} 
Table 2: Global bidder's optimal drop-out price for a sample of valuations under various distributions and synergy levels

\begin{tabular}{|c|c|c|c|c|}
\hline $\begin{array}{c}\text { Global } \\
\text { Bidder's } \\
\text { Valuation } \\
\text { for License A } \\
v_{0 A} \\
\end{array}$ & $\begin{array}{c}\text { Global } \\
\text { Bidder's } \\
\text { Valuation } \\
\text { for License B } \\
v_{0 B} \\
\end{array}$ & $\begin{array}{c}\text { Uniform } \\
\text { Distr. }\end{array}$ & $\begin{array}{c}\text { Beta } \\
\text { Distr. } \\
\text { with } \alpha=5 \\
\text { and } \beta=5 \\
p_{0}^{*}\end{array}$ & $\begin{array}{c}\text { Beta } \\
\text { Distr. } \\
\text { with } \alpha=1 \\
\text { and } \beta=3 \\
p_{0}^{*}\end{array}$ \\
\hline \multicolumn{5}{|c|}{ Synergy =0.2 } \\
\hline 0.25 & 0.2 & 0.2310 & 0.2312 & 0.2610 \\
\hline 0.4 & 0.2 & 0.2641 & 0.2904 & 0.3180 \\
\hline 0.6 & 0.2 & 0.3127 & 0.3712 & 0.3754 \\
\hline 0.8 & 0.2 & 0.3683 & 0.3990 & 0.3982 \\
\hline 0.81 & 0.4 & 0.5591 & 0.5979 & 0.5951 \\
\hline \multicolumn{5}{|c|}{ Synergy=0.4 } \\
\hline 0.25 & 0.2 & 0.2909 & 0.3270 & 0.3507 \\
\hline 0.4 & 0.2 & 0.3528 & 0.4218 & 0.4298 \\
\hline 0.6 & 0.2 & 0.4536 & 0.5412 & 0.5360 \\
\hline 0.8 & 0.2 & 0.5551 & 0.5973 & 0.5940 \\
\hline 0.81 & 0.4 & 0.7325 & 0.7807 & 0.7724 \\
\hline \multicolumn{5}{|c|}{ Synergy =0.6 } \\
\hline 0.25 & 0.2 & 0.3787 & 0.4539 & 0.4576 \\
\hline 0.4 & 0.2 & 0.4667 & 0.5472 & 0.5429 \\
\hline 0.6 & 0.2 & 0.6000 & 0.6653 & 0.6571 \\
\hline 0.8 & 0.2 & 0.7268 & 0.7765 & 0.7680 \\
\hline 0.81 & 0.4 & 0.8733 & 0.8958 & 0.8914 \\
\hline \multicolumn{5}{|c|}{ Synergy =0.8 } \\
\hline 0.25 & 0.2 & 0.5000 & 0.5773 & 0.5714 \\
\hline 0.4 & 0.2 & 0.6000 & 0.6653 & 0.6571 \\
\hline 0.6 & 0.2 & 0.7333 & 0.7791 & 0.7714 \\
\hline 0.8 & 0.2 & 0.8667 & 0.8903 & 0.8857 \\
\hline 0.81 & 0.4 & 1 & 1 & 1 \\
\hline
\end{tabular}


$V C G$ auction is lower than that of the simultaneous ascending auction (SAA).

Proposition 5 a) If the local bidder wins both licenses and the allocation is inefficient, then the revenue of the VCG auction is greater than the revenue of the SAA.

b) If a local bidder wins one license and the global bidder wins the other license without loss, and the allocation is inefficient, then the revenue of the VCG auction is greater than the revenue of the $S A A$ auction. ${ }^{22}$

Unfortunately, the revenue comparison is unclear when the allocation is efficient with the SAA auction. There are cases in which the SAA auction gives higher or lower revenue than the VCG auction in efficient allocations.

Finally, we calculate the expected welfare loss that is created by the SAA auction. We calculated the welfare with the SAA and VCG auction with our code (for 10000 draws). Then, we find the percentage difference between the two auctions' welfare. The results are summarized in Table 3. The expected welfare loss is less than one per cent in all distributions we have used. While this may seem low, the revenue of the 2008 Canadian AWS auction was more than 4 billion Canadian dollars. Even a small percentage decrease in revenue (say 0.5 per cent) might have a relatively big magnitude effect (more than 20 million dollars). The same logic would apply for the welfare.

Table 3: The percentage shortfall in welfare in the SAA relative to the VCG auction under various distributions

\begin{tabular}{cccc}
\hline \hline Synergy & Uniform Distr. & $\begin{array}{c}\text { Beta Distr.with } \alpha=5 \\
\text { and } \beta=5\end{array}$ & $\begin{array}{c}\text { Beta Distr.with } \alpha=1 \\
\text { and } \beta=3\end{array}$ \\
\hline \hline 0.2 & $0.1849 \%$ & $0.2167 \%$ & $0.4643 \%$ \\
0.4 & $0.3103 \%$ & $0.1489 \%$ & $0.5287 \%$ \\
0.6 & $0.2255 \%$ & $0.0375 \%$ & $0.2171 \%$ \\
0.8 & $0.1156 \%$ & $0.0056 \%$ & $0.0771 \%$ \\
\hline \hline
\end{tabular}

${ }^{22}$ We skip the proof of part b since it is similar to part a. 


\section{Revised Model}

In the main model, auction for license B always ends first in equilibrium. We have also studied a revised model in which either auction might end first with a positive probability. ${ }^{23}$ In this model, we assume one local bidder bidding on each license. ${ }^{24}$ To determine the valuations for local bidders $A$ and $B$, we draw two private valuations $v_{1 A}$ and $v_{1 B}$ independently from the distribution function $F($.$) . As in the main model, the global bidder's valuations are drawn$ in pairs from $F$. The maximum and the minimum of these draws become the global bidder's license $A$ and license $B$ valuations, respectively. ${ }^{25}$ Everything else in the main model also applies to the revised model. Since local bidder's valuations are independently drawn, local bidder $B$ 's valuation can be the highest among all bidders. This might happen if local bidder $\mathrm{B}$ is extremely cost efficient in the region it operates (after winning the license).

The main difference between the main model and the revised model is as follows. Since, the local bidder A has a maximum valuation in the main model, the global bidder is more likely to lose license A after winning license B. As a result, in the revised model, the global bidder faces less ex-post loss despite using a higher optimal drop out prices. Hence, the welfare shortfall in the SAA relative to the VCG auction in the revised model is less than the welfare loss in the SAA relative to the VCG auction in the main model.

\section{Conclusion and Discussion}

In this paper, we showed the optimal bidding strategies of global bidders when there are moderate synergies and the licenses are heterogeneous. We also extensively analyzed the inefficient allocation, exposure problem and their effects on revenue.

One natural question to ask is whether there is an optimal mechanism for these types of auctions. Unfortunately, this is a difficult and open problem in the economics and computer

\footnotetext{
${ }^{23}$ The revised model is a technical extension of the main model; hence, we admit that we cannot provide much intuition. The revised model can be requested from the authors.

${ }^{24}$ When we used more than one local bidder, we could not prove that the optimal drop out price of the global bidder will increase as the local bidders drop out. Hence, we could not extend the proof for more than one local bidder case.

${ }^{25}$ This makes the global bidder's license valuations heterogeneous.
} 
science literature since the standard optimal mechanism design techniques cannot be applied when the valuations are multi-dimensional. In our model, the global bidder's valuation is three-dimensional; one valuation for license $A$, one for license $B$ and one for the private synergy parameter. Ulku (2013) tackles multi-dimensional valuations but he essentially reduces the multi-dimensionality into single-dimension, and his techniques are not applicable in our framework. The computer science literature made some progress to find the "almost" optimal mechanism with algorithms (Cai et. al 2011).

We can generalize our main model where there are more A local bidders than B local bidders. For example, if there are more A local bidders, we can draw two valuations for each one of the additional A local bidders from the distribution function $F$ and assign the maximum of the two values as their valuation. This formulation guarantees that $p_{A}>p_{B}$ and our results hold. If there are more B local bidders, with a model where one draws two values and assigns the minimum to the additional B local bidders, $p_{A}<p_{B}$ is possible, ${ }^{26}$ then the global bidder has to deal with cases in which it wins license A first, and our results may not hold. We do not believe that assuming more B local bidders will bring new insights, and we have already analyzed a model where any auction might end with a positive probability.

Our main contribution was our simulations that calculates the probability of inefficient allocations in the SAA auction by using ex-post valuations. We showed that the probability of inefficient allocation can be up to 9 per cent for some distribution functions. To our best knowledge, our paper is the first one calculating these probabilities. We believe that this is a relevant information for Industry Canada, and other policy makers. ${ }^{27}$

Our other contribution is comparing the revenue of the simultaneous ascending auction (SAA) with those of the VCG auction. We find the following relation between the inefficient allocations and the revenue of the auctions. If the valuations are such that the global bidder wins the licenses with an ex-post loss (which is an inefficient outcome), then the revenue of

\footnotetext{
${ }^{26}$ The minimum of two values can be approximately 1 or even exactly 1 , the highest possible value so $p_{A}<p_{B}$ is possible with a positive probability.

${ }^{27}$ Needless to say, more research has to be done to determine the percentage of inefficient allocation since we do not know the exact distribution of valuations. Bajari and Fox (2013) estimate the synergy value to be around 73 per cent of the valuations for one of the US spectrum auctions.
} 
the SAA auction for these valuations would be higher than the revenue of the VCG auction. When local bidders win the license(s) inefficiently (in the SAA auction), the VCG mechanism gives a higher revenue.

In our simulations, there are many cases where the local bidders win inefficiently. We interpret this as a supporting evidence of the exposure problem. The global bidder does not bid high enough from an efficiency point of view, and hence, the local bidders win the licenses inefficiently.

Our simulation code is a complex one so increasing the number of local bidders even to 2 would be a problem for calculating inefficient allocations. However, calculating revenue for the SAA and VCG auction with simulations for more than one local bidder seems more feasible. We are not sure whether such a code will bring new insights.

We use only one global bidder. Albano et. al (2006) writes "In fact, for intermediate values of $\alpha \in(0,1)$ and if $v_{1}$ and $v_{2}$ are different...; showing the existence of a PBE is already problematic in this case." In their paper, $\alpha$ is used to denote synergy and their $v_{1}$ and $v_{2}$ are $v_{0 A}$ and $v_{0 B}$ in our paper, respectively. We use low/moderate synergy at the expense of giving up using more than one global bidder. We also note that Goeree and Lien (2014) uses one global bidder when they assume substitutability of items for local bidders in subsection 4.1 of their paper. Kagel and Levin (2005) is another example where one global bidder is used in the literature. When there are two global bidders, one must write each global bidder's first order conditions separately and solve it simultaneously. The difficulty arises from the fact that while solving these equations, one needs the distribution of the other global bidder's optimal drop out price since $E \Pi_{0}^{1}$ (and $E \Pi_{0}^{2}$ ) will be functions of the other global bidder's optimal drop out price.

\section{Appendix}

\section{Proof of Proposition 1.}

We will prove that there is a unique optimal drop-out price with lemma 6 and lemma 7 below. First we will show that there exists a unique $p_{0}^{*} \in\left(v_{0 B}, \min \left\{v_{0 A}+\theta, 1\right\}\right)$ that solves 
$J\left(p_{0}^{*}\right)=0$. Then, we will prove that only $0, p_{0}^{*}$, and 1 satisfy $F O C=J(p) h(p)=0$. Then, we will show that $p_{0}^{*}$ satisfies the second order condition so it is a local maximum. Then, we will show that 0 and 1 cannot be the maximizer, and, $p_{0}^{*}$ is the unique maximizer.

Lemma 6 There exists a unique $p_{0}^{*}$ such that $J\left(p_{0}^{*}\right)=0$ given the conditions a) to d) in Proposition 3.

Proof of Lemma 6 a). In this case, we have the assumptions of $v_{0 A}+\theta<1$ and $\int_{v_{0 A}}^{v_{0 A}+\theta} G\left(p_{A} \mid v_{0 A}, m_{A}\right) d p_{A}+\left(v_{0 B}-v_{0 A}\right)<0$. We will show that this implies $p_{0}^{*}<v_{0 A}$ (which in turn implies $\left.E \Pi_{0}^{1}>0\right)$.

We have already shown in the text that $J\left(p, m_{A}\right)=E \Pi_{0}^{2}-E \Pi_{0}^{1}$, where $E \Pi_{0}^{2}\left(p, m_{A}\right)=$ $\int_{p}^{v_{0 A}+\theta}\left(v_{0 A}+v_{0 B}+\theta-p-p_{A}\right) g\left(p_{A} \mid p, m_{A}\right) d p_{A}+\int_{v_{0 A}+\theta}^{1}\left(v_{0 B}-p\right) g\left(p_{A} \mid p, m_{A}\right) d p_{A}$ and $E \Pi_{0}^{1}\left(p, m_{A}\right)=$ $\int_{p}^{v_{0 A}}\left(v_{0 A}-p_{A}\right) g\left(p_{A} \mid p, m_{A}\right) d p_{A}$.

To prove uniqueness, we will show that $J\left(p, m_{A}\right)$ is monotonically decreasing from 0 to $v_{0 A}$, and it is positive when $p=v_{0 B}$ and is negative when $p=v_{0 A}$. Hence, there must be a unique root in the interval $v_{0 B}<p<v_{0 A}$.

$$
\begin{gathered}
J\left(p, m_{A}\right)=\int_{p}^{v_{0 A}+\theta}\left(v_{0 A}+v_{0 B}+\theta-p-p_{A}\right) g\left(p_{A} \mid p, m_{A}\right) d p_{A} \\
+\int_{v_{0 A}+\theta}^{1}\left(v_{0 B}-p\right) g\left(p_{A} \mid p, m_{A}\right) d p_{A}-\int_{p}^{v_{0 A}}\left(v_{0 A}-p_{A}\right) g\left(p_{A} \mid p, m_{A}\right) d p_{A}
\end{gathered}
$$

By using $\int_{p}^{1} g\left(p_{A} \mid p, m_{A}\right) d p_{A}=1$ since $g\left(p_{A} \mid p, m_{A}\right)$ is a probability density function on the support $[p, 1]$, we have $\left(v_{0 B}-p\right) \int_{p}^{1} g\left(p_{A} \mid p, m_{A}\right) d p_{A}=v_{0 B}-p$, we can re-write it as

$$
J\left(p, m_{A}\right)=-\int_{p}^{v_{0 A}}\left(v_{0 A}-p_{A}\right) g\left(p_{A} \mid p, m_{A}\right) d p_{A}+\int_{p}^{v_{0 A}+\theta}\left(v_{0 A}+\theta-p_{A}\right) g\left(p_{A} \mid p, m_{A}\right) d p_{A}+
$$
$\left(v_{0 B}-p\right)$

We will use integration by parts, $\int u d v=u v-\int v d u$, twice in what follows to re-write $J($.$) .$

First we assume that $u=v_{0 A}-p_{A}$ and $v=G\left(p_{A} \mid p, m_{A}\right)$; then assume that $u=v_{0 A}+\theta-p_{A}$ and $v=G\left(p_{A} \mid p, m_{A}\right)$, we have

$$
\begin{aligned}
& J\left(p, m_{A}\right)=-\left.\left(v_{0 A}-p_{A}\right) G\left(p_{A} \mid p, m_{A}\right)\right|_{p} ^{v_{0 A}}+\int_{p}^{v_{0 A}} G\left(p_{A} \mid p, m_{A}\right) d\left(v_{0 A}+p_{A}\right) \\
+ & \left.\left(v_{0 A}-\theta-p_{A}\right) G\left(p_{A} \mid p, m_{A}\right)\right|_{p} ^{v_{0 A}+\theta}-\int_{p}^{v_{0 A}+\theta} G\left(p_{A} \mid p, m_{A}\right) d\left(v_{0 A}+\theta-p_{A}\right)+\left(v_{0 B}-p\right) \\
= & -\int_{p}^{v_{0 A}} G\left(p_{A} \mid p, m_{A}\right) d p_{A}+\int_{p}^{v_{0 A}+\theta} G\left(p_{A} \mid p, m_{A}\right) d p_{A}+\left(v_{0 B}-p\right) \\
= & \int_{v_{0 A}}^{v_{0 A}+\theta} G\left(p_{A} \mid p, m_{A}\right) d p_{A}+\left(v_{0 B}-p\right)
\end{aligned}
$$


We take partial derivative of $J\left(p, m_{A}\right)$ with respect to $p$ :

$\frac{\partial J\left(p, m_{A}\right)}{\partial p}=\frac{\partial}{\partial p}\left[\int_{v_{0 A}}^{v_{0 A}+\theta} G\left(p_{A} \mid p, m_{A}\right) d p_{A}\right]-1$. We will prove that this is negative since the term $\frac{\partial}{\partial p}\left[\int_{v_{0 A}}^{v_{0 A}+\theta} G\left(p_{A} \mid p, m_{A}\right) d p_{A}\right]$ is negative. The term inside is non-negative since it is a cumulative distribution function. We must show that $\frac{\partial G\left(p_{A} \mid p, m_{A}\right)}{\partial p} \leq 0$ to prove this. While one can easily see that this is correct (as p increases the cumulative distribution conditional on p decreases), we will give a formal proof by using Leibniz's rule.

$$
\begin{aligned}
& \Leftrightarrow \frac{\partial G\left(p_{A} \mid p, m_{A}\right)}{\partial p}=\frac{\partial\left[\left(\frac{\int_{p}^{p_{A}} f(v) d v}{\int_{p}^{1} f(v) d v}\right)^{m_{A}}\right]}{\partial p} \\
&=-\left(m_{A}\right) f(p) \frac{\left(\int_{p}^{p_{A}} f(v) d v\right)^{m_{A}-1}}{\left(\int_{p}^{1} f(v) d v\right)^{m}}+\left(m_{A}\right) f(p) \frac{\left(\int_{p}^{p_{A}} f(v) d v\right)^{m_{A}}}{\left(\int_{p}^{1} f(v) d v\right)^{m_{A}+1}} \\
&=\frac{\left(m_{A}\right) f(p)\left(\int_{p}^{p_{A}} f(v) d v\right)^{m_{A}-1}}{\left(\int_{p}^{1} f(v) d v\right)^{m}}\left[-1+\frac{\int_{p}^{p_{A}} f(v) d v}{\int_{p}^{1} f(v) d v}\right] \\
&=\frac{\left(m_{A}\right) f(p)\left(\int_{p}^{p_{A}} f(v) d v\right)^{m_{A}-1}}{\left(\int_{p}^{1} f(v) d v\right)^{m_{A}}}\left[-1+F\left(p_{A} \mid p, m_{A}\right)\right]<0 \quad\left(\leq 0 \text { only if } p_{A}=1\right) .
\end{aligned}
$$

Thus, $J\left(p, m_{A}\right)$ is a monotonically decreasing function of $p \in\left(0, v_{0 A}\right)$.

If $p=v_{0 B}$, then $J\left(p=v_{0 B}, m_{A}\right)=-\int_{v_{0 B}}^{v_{0 A}} G\left(p_{A} \mid v_{0 B}, m_{A}\right) d p_{A}+\int_{v_{0 B}}^{v_{0 A}+\theta} G\left(p_{A} \mid v_{0 B}, m_{A}\right) d p_{A}$ $=\int_{v_{0 A}}^{v_{0 A}+\theta} G\left(p_{A} \mid v_{0 B}, m_{A}\right) d p_{A}>0$.

If $p=v_{0 A}, J\left(p=v_{0 A}, m_{A}\right)=0+\int_{v_{0 A}}^{v_{0 A}+\theta} G\left(p_{A} \mid v_{0 A}, m_{A}\right) d p_{A}+\left(v_{0 B}-v_{0 A}\right)<0$ by our assumption.

Hence, there is a unique root, $p_{0}^{*}$, such that $v_{0 B}<p_{0}^{*}<v_{0 A}$.

b) In this case, we have the assumptions of $v_{0 A}+\theta<1$ and $\int_{v_{0 A}}^{v_{0 A}+\theta} G\left(p_{A} \mid v_{0 A}, m_{A}\right) d p_{A}+$ $\left(v_{0 B}-v_{0 A}\right) \geq 0$ which we will show that this implies $p_{0}^{*} \geq v_{0 A}$. And this condition in turn implies that $E \Pi_{0}^{1}=0$, since the global bidder drops from both licenses. $E \Pi_{0}^{2}\left(p, m_{A}\right)=$ $J\left(p, m_{A}\right)=\int_{p}^{v_{0 A}+\theta}\left(v_{0 A}+v_{0 B}+\theta-p-p_{A}\right) g\left(p_{A} \mid p, m_{A}\right) d p_{A}+\int_{v_{0 A}+\theta}^{1}\left(v_{0 B}-p\right) g\left(p_{A} \mid p, m_{A}\right) d p_{A}=$ $\int_{p}^{v_{0 A}+\theta} G\left(p_{A} \mid p, m_{A}\right) d p_{A}+\left(v_{0 B}-p\right)$.

When $p \geq v_{0 A}$, we take partial derivative of $J\left(p, m_{A}\right)$ with respect to $p$, we have,

$$
\frac{\partial J\left(p, m_{A}\right)}{\partial p}=\frac{\partial}{\partial p}\left[\int_{p}^{v_{0 A}+\theta} G\left(p_{A} \mid p, m_{A}\right) d p_{A}\right]-1=-G\left(p_{A} \mid p, m_{A}\right)+\int_{p}^{v_{0 A}+\theta} \frac{\partial G\left(p_{A} \mid p, m_{A}\right)}{\partial p} d p_{A}-1<0,
$$
since $\frac{\partial G\left(p_{A} \mid p, m_{A}\right)}{\partial p}<0$.

Thus, $J\left(p, m_{A}\right)$ is a monotonically decreasing function of $p$ in $\left(v_{0 A}, 1\right)$.

Our assumption $\int_{v_{0 A}}^{v_{0 A}+\theta} G\left(p_{A} \mid v_{0 A}, m_{A}\right) d p_{A}+\left(v_{0 B}-v_{0 A}\right) \geq 0$ implies that $J\left(p=v_{0 A}, m_{A}\right) \geq$ 0 . If $p=v_{0 A}+\theta$, then $J\left(p=v_{0 A}+\theta, m_{A}\right)=\left(v_{0 B}-v_{0 A}-\theta\right)<0$. Thus, there is a unique solution, $p_{0}^{*}$, in the interval $\left[v_{0 A}, v_{0 A}+\theta\right)$. 
c) In this case, we have the assumptions of $v_{0 A}+\theta \geq 1$ and $\int_{v_{0 A}}^{1} G\left(p_{A} \mid v_{0 A}, m_{A}\right) d p_{A}+\left(v_{0 B}+\right.$ $\theta-1)<0$ which implies $v_{0 A}<p_{0}^{*}$. And this condition in turn implies that $E \Pi_{0}^{1}>0$. That is, $E \Pi_{0}^{2}\left(p, m_{A}\right)=\int_{p}^{1}\left(v_{0 A}+v_{0 B}+\theta-p-p_{A}\right) g\left(p_{A} \mid p, m_{A}\right) d p_{A}$ and $E \Pi_{0}^{1}\left(p, m_{A}\right)=\int_{p}^{v_{0 A}}\left(v_{0 A}-\right.$ $\left.p_{A}\right) g\left(p_{A} \mid p, m_{A}\right) d p_{A}$.

$J\left(p, m_{A}\right)=E \Pi_{0}^{2}\left(p, m_{A}\right)-E \Pi_{0}^{1}\left(p, m_{A}\right)=\int_{p}^{1}\left(v_{0 A}+v_{0 B}+\theta-p-p_{A}\right) g\left(p_{A} \mid p, m_{A}\right) d p_{A}-$ $\int_{p}^{v_{0 A}}\left(v_{0 A}-p_{A}\right) g\left(p_{A} \mid p, m_{A}\right) d p_{A}$

Similar to part a, we use integration by parts twice. First, we assume that $u=v_{0 A}-p_{A}$ and $v=G\left(p_{A} \mid p, m_{A}\right)$ for the first integral; then assume that $u=v_{0 A}+v_{0 B}+\theta-p-p_{A}$ and $v=G\left(p_{A} \mid p, m_{A}\right)$ for the second integral. As a result,

$$
\begin{aligned}
& \quad J\left(p, m_{A} \mid v_{0 A}+\theta>1\right)=-\left.\left(v_{0 A}-p_{A}\right) G\left(p_{A} \mid p, m_{A}\right)\right|_{p} ^{v_{0 A}}-\int_{p}^{v_{0 A}} G\left(p_{A} \mid p, m_{A}\right) d p_{A}+\left(v_{0 A}+\right. \\
& \left.v_{0 B}+\theta-p-p_{A}\right)\left.G\left(p_{A} \mid p, m_{A}\right)\right|_{p} ^{1}+\int_{p}^{1} G\left(p_{A} \mid p, m_{A}\right) d p_{A} \\
& =-\int_{p}^{v_{0 A}} G\left(p_{A} \mid p, m_{A}\right) d p_{A}+\left(v_{0 A}+v_{0 B}+\theta-p-1\right)+\int_{p}^{1} G\left(p_{A} \mid p, m_{A}\right) d p_{A} \\
& =\left(v_{0 A}+v_{0 B}+\theta-p-1\right)+\int_{v_{0 A}}^{1} G\left(p_{A} \mid p, m_{A}\right) d p_{A}
\end{aligned}
$$

We take partial derivative of $J\left(p, m_{A}\right)$ with respect to $p$, we have,

$$
\frac{\partial J\left(p, m_{A}\right)}{\partial p}=\frac{\partial}{\partial p}\left[\int_{v_{0 A}}^{1} G\left(p_{A} \mid p, m_{A}\right)\right]-1<0
$$

It is negative since the term $\frac{\partial}{\partial p}\left[\int_{v_{0 A}}^{1} G\left(p_{A} \mid p, m_{A}\right)\right]$ is negative. And we have already shown that $\frac{\partial G\left(p_{A} \mid p, m_{A}\right)}{\partial p} \leq 0$. Thus, $J\left(p, m_{A}\right)$ is monotonically decreasing function of $p$ in $\left(0, v_{0 A}\right)$.

If $p=v_{0 B}$, then $J\left(p=v_{0 B}, m_{A}\right)=\int_{v_{0 A}}^{1} G\left(p_{A} \mid v_{0 B}, m_{A}\right) d p_{A}+\left(v_{0 A}+\theta-1\right)>0$ since $v_{0 A}+\theta>1$.

If $p=v_{0 A}, J\left(p=v_{0 A}, m_{A}\right)=0+\int_{v_{0 A}}^{1} G\left(p_{A} \mid v_{0 A}, m_{A}\right) d p_{A}+\left(v_{0 B}+\theta-1\right)<0$ by our assumption.

Hence, there is a unique root in the interval $v_{0 B}<p_{0}^{*}<v_{0 A}$.

d) In this case, we have the assumptions of $v_{0 A}+\theta \geq 1$ and $\int_{v_{0 A}}^{1} G\left(p_{A} \mid v_{0 A}, m_{A}\right) d p_{A}+$ $\left(v_{0 B}+\theta-1\right) \geq 0$ which implies $p_{0}^{*} \geq v_{0 A}$. And this condition in turn implies that $E \Pi_{0}^{1}=0$, and $E \Pi_{0}^{2}\left(p, m_{A}\right)=J\left(p, m_{A}\right)=\int_{p}^{1}\left(v_{0 A}+v_{0 B}+\theta-p-p_{A}\right) g\left(p_{A} \mid p, m_{A}\right) d p_{A}$ $=\int_{p}^{1} G\left(p_{A} \mid p, m_{A}\right) d p_{A}+\left(v_{0 A}+v_{0 B}+\theta-p-1\right)$.

When $p>v_{0 A}$, we take partial derivative of $J\left(p, m_{A}\right)$ with respect to $p$, we have, $\frac{\partial J\left(p, m_{A}\right)}{\partial p}=\frac{\partial}{\partial p}\left[\int_{p}^{1} G\left(p_{A} \mid p, m_{A}\right) d p_{A}\right]-1<0$, since $\frac{\partial G\left(p_{A} \mid p, m_{A}\right)}{\partial p}<0$. 
Thus, $J\left(p, m_{A}\right)$ is monotonically decreasing function of $p$ in $\left(v_{0 A}, 1\right)$.

Our assumption $\int_{v_{0 A}}^{1} G\left(p_{A} \mid v_{0 A}, m_{A}\right) d p_{A}+\left(v_{0 B}+\theta-1\right) \geq 0$ implies that $J\left(p=v_{0 A}, m_{A}\right) \geq$ 0 . If $p=1$, then $J\left(p=1, m_{A}\right)=\left(v_{0 B}+v_{0 A}+\theta-2\right)<0$. Since $v_{0 B}+v_{0 A}+\theta<2$ by our average valuation is less than 1 assumption in the proposition. Thus, there is a unique solution, $p_{0}^{*}$, in the interval $\left[v_{0 A}, 1\right)$.

Lemma 7 a) Only 0,1, and $p_{0}^{*}$ solves the FOC.

b) $p_{0}^{*}$ satisfies the second order condition.

c) 0 and 1 cannot maximize the expected payoff.

d) $\frac{d p_{0}^{*}}{d m_{A}}<0$. That is, when the number of active firms in license A auction decreases, the optimal drop-out price will increase.

Proof a) From lemma 6, we can write the $F O C=J\left(p, m_{A}\right) h(p)$ with the abuse of notation since $J\left(p, m_{A}\right)$ takes different forms in part a to d. It is easy to see that FOC is equal to zero only if $J\left(p, m_{A}\right)=0$ or $h(p)=0$. In lemma 6 , we showed that there is a unique $p_{0}^{*} \in\left(v_{0 B}, \min \left\{v_{0 A}+\theta, 1\right\}\right)$ that makes $J\left(p, m_{A}\right)=0$. Hence, $F O C\left(p_{0}^{*}\right)=0$. This proves $p_{0}^{*}$ solves FOC.

Now, we will show that only $p=0$ or $p=1$ solves $h(p)=0$. Since $H(p)=[1-(1-$ $\left.F(p))^{2}\right]^{m_{B}}$, we have $h(p)=2 m_{B}(1-F(p)) f(p)\left[1-(1-F(p))^{2}\right]^{m_{B}-1}$. Then $h(p)=0$ only if $1-F(p)=0$ which implies $p=1$, or $1-(1-F(p))^{2}=0$ which implies $p=0$. There is no $p \in(0,1)$ that makes $h(p)=0$ since by our assumption $f(p)>0$ when $p \in(0,1]$ and $m_{B} \geq 1$.

b) $F O C=J\left(p, m_{A}\right) h(p)$, hence $S O C=J^{\prime}\left(p, m_{A}\right) h(p)+J\left(p, m_{A}\right) h^{\prime}(p)$. When $p=p_{0}^{*}$, $J\left(p_{0}^{*}\right)=0$ as shown in lemma 6 , hence, $S O C=J^{\prime}\left(p_{0}^{*}, m_{A}\right) h\left(p_{0}^{*}\right)$. This is negative since we have showed in lemma 6 that $J\left(p, m_{A}\right)$ is a decreasing function and $h(p)>0$ for any $p \in(0,1)$.

c) While it is straightforward to see that dropping out at $p=0$ cannot be optimal, we will provide the proof. When we consider $p=0$, the expected payoff function is written as in 
part a and $\mathrm{c}$ of Proposition 1 since the optimal drop out price is assumed to be $p=0<v_{0 A}$. We have shown that $J(p)$ is a decreasing function in $\left(0, v_{0 A}\right)$ and $J\left(p_{0}^{*}\right)=0$. These two facts imply that that $J(p)>0$ for any $p \in\left(0, p_{0}^{*}\right)$. Since $h(p)>0$ for $p \in(0,1)$, we have $F O C=J(p) h(p)>0$ for any $p \in\left(0, p_{0}^{*}\right)$. Since $J(p)$ and $h(p)$ are continuous, FOC $>0$ implies that the global bidder can increase its payoff by bidding higher than 0 . Hence, 0 cannot be the maximizer. One can similarly prove that $p=1$ cannot be the maximizer by using part $\mathrm{b}$ and $\mathrm{d}$ of Proposition 1. Therefore $p_{0}^{*}$ is the only price that satisfies the FOC and SOC and it is unique.

d) Next, we show that as the number of active firms in license A auction decreases, the optimal drop-out price will increase. We will use the implicit function theorem for this: ${ }^{28}$

$$
\Leftrightarrow \frac{d p_{0}^{*}}{d m_{A}}=-\frac{\frac{\partial F O C\left(p_{0}^{*}, m_{A}\right)}{\partial m_{A}}}{\frac{\partial F O C\left(p_{0}^{*}, m_{A}\right)}{\partial p_{0}^{*}}}<0 .
$$

We have $\frac{\partial F O C\left(p_{0}^{*}, m_{A}\right)}{\partial p_{0}^{*}}=J^{\prime}\left(p_{0}^{*}\right) h\left(p_{0}^{*}\right)+J\left(p_{0}^{*}\right) h^{\prime}\left(p_{0}^{*}\right)=J^{\prime}\left(p_{0}^{*}\right) h\left(p_{0}^{*}\right)<0$. The equality holds since $J\left(p_{0}^{*}\right)=0$, and the inequality holds since we have proved in lemma 6 that $J^{\prime}\left(p_{0}^{*}\right)<0$.

By using Leibniz's rule for differentiation under the integral sign, we take partial derivative of $F O C\left(p_{0}^{*}, m_{A}\right)=\left[\int_{v_{0 A}}^{v_{0 A}+\theta} G\left(p_{A} \mid p_{0}^{*}, m_{A}\right) d p_{A}+\left(v_{0 B}-p_{0}^{*}\right)\right] h\left(p_{0}^{*}\right)$ with respect to $m_{A}$, since $h\left(p_{0}^{*}\right)>0$ and $\frac{\partial h\left(p_{0}^{*}, m_{B}\right)}{\partial m_{A}}=0$, then we have,

$$
\frac{\partial F O C\left(p_{0}^{*}, m_{A}\right)}{\partial m_{A}}=\left[\int_{v_{0 A}}^{v_{0 A}+\theta} \frac{\partial G\left(p_{A} \mid p_{0}^{*}, m_{A}\right)}{\partial m_{A}} d p_{A}\right] h\left(p_{0}^{*}\right)=\left[\int_{v_{0 A}}^{v_{0 A}+\theta} \ln \left(F\left(p_{A} \mid p, m_{A}\right)\right) G\left(p_{A} \mid p_{0}^{*}, m_{A}\right) d p_{A}\right] h\left(p_{0}^{*}\right)<
$$
0 , since $\frac{\partial G\left(p_{A} \mid p_{0}^{*}, m_{A}\right)}{\partial m_{A}}=\ln \left(F\left(p_{A} \mid p_{0}^{*}, m_{A}\right)\right) G\left(p_{A} \mid p, m_{A}\right)<0$. Hence, we show that $\frac{\partial F O C\left(p_{0}^{*}, m_{A}\right)}{\partial m_{A}}<$ 0 holds.

$$
\text { Since } \frac{\partial F O C\left(p_{0}^{*}, m_{A}\right)}{\partial p_{0}^{*}}<0 \text { and } \frac{\partial F O C\left(p_{0}^{*}, m_{A}\right)}{\partial m_{A}}<0 \text {, we have, } \frac{d p_{0}^{*}}{d m_{A}}=-\frac{\frac{\partial F O C\left(p_{0}^{*}, m_{A}\right)}{\partial m_{A}}}{\frac{\partial F O C\left(p_{0}^{*}, m_{A}\right)}{\partial p_{0}^{*}}}<0
$$

By the implicit function theorem, we show that the optimal drop-out price increases as the number of local firms, $m_{A}$, decreases.

Proof of Lemma 3. If the global bidder wins only one license and has an ex-post loss, then clearly $p_{0}^{*}>p_{B}>v_{0 B}$ must hold since the global bidder will wait until the price reaches $p_{0}^{*} \cdot{ }^{29}$ To prove the inefficiency for this case, we will use the inequalities $v_{0 B}<p_{B}$

\footnotetext{
${ }^{28}$ We treat $m_{A}$ as a continuous variable here although it is discrete. We do not see any harm in doing this since the function is continuous in $m_{A}$ and the result will be valid even when $m_{A}$ is discrete.

${ }^{29}$ Note that the global bidder cannot win license $A$ first since local bidders' minimum valuations are
} 
and $v_{0 A}+\theta<p_{A}$. The latter one holds since the global bidder does not win license $A$. This immediately shows that we have $v_{0 A}+v_{0 B}+\theta<p_{A}+p_{B}$; the efficient outcome is that the local bidders must have won both licenses. Hence, the outcome is inefficient.

If the global bidder wins two licenses, winning license $B$ implies $p_{0}^{*}>p_{B}$ and winning license $A$ implies $p_{A}<v_{0 A}+\theta$. The global bidder makes a loss by our assumption, that is $v_{0 A}+v_{0 B}+\theta-p_{A}-p_{B}<0$ which implies $v_{0 B}-p_{B}<p_{A}-v_{0 A}-\theta<0$. Hence, we have $v_{0 B}<p_{B}$. Hence, we showed that $p_{0}^{*}<v_{0 B}<p_{B}$. The loss condition implies $v_{0 A}+v_{0 B}+\theta<p_{A}+p_{B}$ which proves that the outcome of global bidder winning two licenses is inefficient since local bidders must have won the licenses.

Proof of Proposition 4. When the global bidder wins the license(s) with an ex-post loss, then the revenue of VCG auction, $R_{V C G}$, is lower than the revenue of SAA, $R_{S A A}$. We know that $p_{B}>v_{0 B}$ by lemma 3. There are two cases that we must consider, (i) global bidder wins both licenses with an ex-post loss and (ii) global bidder wins only license B with an ex-post loss. We have already explained that global bidder cannot win license A only and makes a loss since it will bid like a local bidder after losing license B.

i) The revenue of SAA auction is $R_{S A A}=p_{A}+p_{B}$, and since the global bidder wins both licenses and makes a loss, we have $p_{A}+p_{B}>v_{0 A}+v_{0 B}+\theta$. We know that $v_{0 A}+\theta>p_{A}$ since global bidder wins license A and B in the SAA auction. In a VCG auction, the local bidders will win the licenses if $v_{0 A}<p_{A}$. The payment of local bidder $B$ can be calculated as follows. If local bidder $\mathrm{B}$ does not participate in the auction, the welfare of others (in the efficient allocation) is $p_{A}+v_{0 B}$ since local bidder $\mathrm{A}$ will win license $\mathrm{A}$ and global bidder will win license B. If local bidder B participates in the auction, the welfare of others (in the efficient allocation) will be $p_{A}$. The difference is the payment of local bidder $\mathrm{B}$ which is $v_{0 B}$. A symmetric calculation will show that the local bidder A's payment will be $v_{0 A}$. Hence, $R_{V C G}=v_{0 A}+v_{0 B}<R_{S A A}=p_{A}+p_{B}$ by the global bidder's loss condition and that $\theta>0$.

If $v_{0 A}>p_{A}$, then the efficient allocation is that global bidder wins license $\mathrm{A}$ and local bidder $\mathrm{B}$ wins license $\mathrm{B}$. The global bidder's payment will be $p_{A}$ since if the global bidder assigned to $B$ and maximum valuations are assigned to $A$. 
does not participate in the auction, the welfare of others in the efficient allocation is $p_{A}+p_{B}$ but if it participates, it is only $p_{B}$. The difference is $p_{A}$. The payment of local bidder $\mathrm{B}$ is $v_{0 B}+\theta$ since if local bidder $\mathrm{B}$ does not participate in the auction, the welfare of others in the efficient allocation is $v_{0 A}+v_{0 B}+\theta$, and if it participates, the welfare of others is $v_{0 A}$. The difference is the payment of local bidder B. $R_{V C G}=p_{A}+v_{0 B}+\theta<R_{S A A}=p_{A}+p_{B}$. The inequality follows since the global bidder's loss condition $v_{0 A}+v_{0 B}+\theta<p_{A}+p_{B}$ can be re-written as $v_{0 A}-p_{A}<p_{B}-\left(v_{0 B}+\theta\right)$ but this is positive since $v_{0 A}>p_{A}$ by our assumption. This implies $v_{0 B}+\theta<p_{B}$ and $R_{V C G}=p_{A}+v_{0 B}+\theta<R_{S A A}=p_{A}+p_{B}$.

(ii) Since the global bidder will pay $p_{B}$ for license B, and local bidder A will pay $v_{0 A}+\theta$ for license $\mathrm{A}$, the revenue of the SAA auction, $R_{S A A}=v_{0 A}+\theta+p_{B}$ in the case when $p_{A} \geq p_{B}$. The VCG auction will result in an efficient auction; hence, local bidders will win each license. The payment of local bidder B is calculated as follows. If local bidder B does not participate in the auction, the welfare of others is $p_{A}+v_{0 B}$ since we know that $p_{A}>v_{0 A}+\theta$ by the fact that global bidder lost license $\mathrm{A}$ in the SAA auction. When local bidder $\mathrm{B}$ participates in the auction, the welfare of the others is $p_{A}$. Hence, the payment of local bidder $\mathrm{B}$ is $v_{0 B}$. The payment of local bidder $\mathrm{A}$ is calculated as follows. If local bidder A does not participate in the auction, the welfare of others is $v_{0 A}+v_{0 B}+\theta$ if $v_{0 B}+\theta>p_{B}$. If $v_{0 B}+\theta<p_{B}$, then it is, $v_{0 A}+p_{B}$. If local bidder $\mathrm{A}$ participates in the auction, then the welfare of others is $p_{B}$. Therefore $R_{V C G}=v_{0 A}+v_{0 B}+\theta-p_{B}+v_{0 B}$ if $v_{0 B}+\theta>p_{B}$. $R_{V C G}=v_{0 A}+v_{0 B}+\theta+\left(v_{0 B}-p_{B}\right)<R_{S A A}=v_{0 A}+\theta+p_{B}$ since $v_{0 B}<p_{B}$ by Lemma 3.

If $v_{0 B}+\theta<p_{B}$, then local bidder A's payment is $v_{0 A}$. Since $\theta>0$ and $p_{B}>v_{0 B}$ by Lemma $3, R_{V C G}=v_{0 A}+v_{0 B}<R_{S A A}=v_{0 A}+\theta+p_{B}$.

Symmetrically, when the global bidder will pay $p_{A}$ for license $\mathrm{B}$, and local bidder B will pay $v_{0 B}+\theta$ for license $\mathrm{B}$, the revenue of the SAA auction, $R_{S A A}=v_{0 B}+\theta+p_{A}$ in the case when $p_{A}<p_{B}$. The VCG auction will result in an efficient auction; hence, local bidders will win each license. The payment of local bidder $\mathrm{A}$ is calculated as follows. If local bidder A does not participate in the auction, the welfare of others is $p_{B}+v_{0 A}$ since we know that $p_{B}>v_{0 B}+\theta$ by the fact that global bidder lost license B in the SAA auction. When local 
bidder A participates in the auction, the welfare of the others is $p_{A}$. Hence, the payment of local bidder $\mathrm{B}$ is $v_{0 B}$. The payment of local bidder $\mathrm{A}$ is calculated as follows. If local bidder A does not participate in the auction, the welfare of others is $v_{0 A}+v_{0 B}+\theta$ if $v_{0 A}+\theta>p_{A}$. If $v_{0 A}+\theta<p_{A}$, then it is, $v_{0 B}+p_{A}$. If local bidder $\mathrm{B}$ participates in the auction, then the welfare of others is $p_{A}$. Therefore $R_{V C G}=v_{0 A}+v_{0 B}+\theta-p_{A}+v_{0 A}$ if $v_{0 A}+\theta>p_{A}$. $R_{V C G}=v_{0 A}+v_{0 B}+\theta+\left(v_{0 A}-p_{A}\right)<R_{S A A}=v_{0 B}+\theta+p_{A}$ since $v_{0 A}<p_{A}$.

If $v_{0 A}+\theta<p_{A}$, then local bidder B's payment is $v_{0 B}$. Since $\theta>0$ and $p_{A}>v_{0 A}$, $R_{V C G}=v_{0 A}+v_{0 B}<R_{S A A}=v_{0 B}+\theta+p_{A}$.

This completes the proof of Proposition 4.

Proof of Proposition 5. a) Each local bidder wins a license in the SAA auction; hence, we know that $v_{0 A}<p_{A}$ and $v_{0 B}<p_{0}^{*}<p_{B}$. We also know that the allocation is not efficient by assumption; hence, we must have $p_{A}+p_{B}<v_{0 A}+v_{0 B}+\theta .{ }^{30} R_{S A A}=v_{0 A}+p_{0}^{*}$.

If the global bidder obtains both licenses in the VCG auction, it is easy to calculate that the auction revenue is $R_{V C G}=p_{A}+p_{B}>R_{S A A}=v_{0 A}+p_{0}^{*}$.

b) The global bidder wins only license $\mathrm{A}$ and local bidder wins license $\mathrm{B}$ and the global bidder makes no loss. ${ }^{31}$ Then $R_{S A A}=p_{0}^{*}+p_{A}$. We also know that $v_{0 A}>p_{A}$ and $p_{B}>$ $p_{0}^{*}>v_{0 B}$ from the result of the SAA auction. Hence, in an efficient outcome the global bidder must obtain license A so the only efficient outcome must be global bidder winning both licenses which implies $v_{0 A}+v_{0 B}+\theta>v_{0 A}+p_{B}$ given that SAA outcome is inefficient. But then since the global bidder will win both licenses, $R_{V C G}=p_{A}+p_{B}>R_{S A A}=p_{0}^{*}+p_{A}$ since $p_{B}>p_{0}^{*}$.

This completes the proof of Proposition 5.

\footnotetext{
${ }^{30}$ Local bidder $\mathrm{A}$ winning the license $\mathrm{A}$ and the global bidder winning license $\mathrm{B}$ cannot be an efficient allocation given the inequalities

${ }^{31}$ If the global bidder wins license B with no loss and local bidder wins license A, then the outcome cannot be inefficient since $p_{B}<v_{0 B}$ must be satisfied by the no-loss condition, and $p_{A}>v_{0 A}+\theta$ condition must be satisfied by the fact that global bidder does not win license A but this is an efficient outcome.
} 


\section{References}

[1] Albano, G.L., F. Germano, S. Lovo. 2001. A comparison of standard multi-unit auctions with synergies. Econom. Lett. 71(10) 55-60.

[2] Albano, G.L., F. Germano, S. Lovo. 2006. Ascending auctions for multiple objects: the case for the Japanese design. Econom. Theory 28 331-355.

[3] Ausubel, L.M., P. Milgrom. 2006. The lovely but lonely Vickrey auction. Peter Cramton, Yoav Shoham, Richard Steinberg, eds. Combinatorial Auctions. MIT Press, Cambridge, MA.

[4] Bajari, P. and J. Fox. 2013. Measuring the efficiency of an FCC spectrum auction. American Economic Journal: Microeconomics 5 100-146.

[5] Brusco, S., G. Lopomo. 2002. Collusion via signaling in simultaneous ascending bid auctions with heterogeneous objects, with and without complementarities. Rev. Econom. Stud. 69 407-463.

[6] Brusco, S., G. Lopomo. 2009. Simultaneous ascending auctions with complementarities and known budget constraints. Econom. Theory 38 105-124.

[7] Chakraborty, I. 2004. Multi-unit auctions with synergy. Econom. Bulletin 4 1-14.

[8] Cai, Y., C. Daskalakis, S. M. Weinberg. 2011. On Optimal Multidimensional Mechanism Design. Newsletter of the ACM Special Interest Group on E-commerce. 10(2).

[9] Cramton, P., J. Schwartz. 2000. Collusive Bidding: Lessons from the FCC Spectrum Auctions. Journal of Regulatory Economics 17 229-252.

[10] Englmaier, F., P. Guillen, L. Llorente, S. Onderstal, R. Sausgruber. 2009. The chopstick auction: a study of the exposure problem in multi-unit auctions. Internat. J. Indust. Organ. 27 286-291. 
[11] Goeree, J.K., Lien Y. 2014. An equilibrium analysis of the simultaneous ascending auction. Journal of Economic Theory 153 506-533.

[12] Kagel, J.H., D. Levin. 2005. Multi-unit demand auctions with synergies: behavior in sealed-bid versus ascending-bid uniform-price auctions. Games Econom. Behav. 53 170207.

[13] Katok, E., A.E. Roth. 2004. Auctions of homogeneous goods with increasing returns: xxperimental comparison of alternative Dutch auctions. Management Sci. 50 1044-1063.

[14] Krishna, V., R.W. Rosenthal. 1996. Simultaneous auctions with synergies. Games Econom. Behav. 17 1-31.

[15] Mishra, D., D.C. Parkes. 2009. Multi-item Vickrey-Dutch auctions. Games Econom. Behav. 66 326-347.

[16] Menicucci, D. 2003. Optimal two-object auctions with synergies. Rev. Econom. Design 8 143-164.

[17] Porter, D.P. 1999. The effect of bid withdrawal in a multi-object auction. Rev. Econom. Design 4 73-97.

[18] Rosenthal, R.W., R. Wang. 1996. Simultaneous auctions with synergies and common values. Games Econom. Behav. 17 32-55.

[19] Ulku, L. 2013. Optimal combinatorial mechanism design. Economic Theory. 53 473-498

[20] Wang, R. 2000. Bidding and renegotiation in procurement auctions. Eur. Econom. Rev. 44 1577-1597.

\section{Online Appendix: Auction $A$ Ending First with a Positive Probability}

In this section, we revise the model so that either auction might end first with a positive probability. We assume one local bidder bidding on each license. To determine the valuations 
for local bidders $A$ and $B$, we draw two private valuations $v_{1 A}$ and $v_{1 B}$ independently from the distribution function $F($.$) . As in the main model, the global bidder's valuations are$ drawn in pairs from $F$. The maximum and the minimum of these draws become the global bidder's license $A$ and license $B$ valuations, respectively. ${ }^{32}$ Everything else in the main model also applies to the current model. Since local bidder's valuations are independently drawn, local bidder $B$ 's valuation can be the highest among all bidders; as a result, either auction can end with a positive probability.

It is straightforward to see that the local bidder's equilibrium strategy, and the global bidder's equilibrium strategy after winning the first license are the same as in the original model. The only change is the optimal drop out price for the global bidder since the global bidder might win license $A$ or $B$ first.

To calculate the optimal drop-out price for the global bidder when his average valuation is less than 1, one should maximize the global bidder's expected payoff. The expected payoff for the global bidder is:

$$
\begin{array}{r}
\max _{p} \operatorname{Pr}\left(v_{1 A} \geq v_{1 B}\right)\left[\int _ { p _ { c } } ^ { p } \left[\int_{v_{1 B}}^{\min \left\{v_{0 A}+\theta, 1\right\}}\left(v_{0 A}+\theta-v_{1 A}+v_{0 B}-v_{1 B}\right) d F\left(v_{1 A} \mid v_{1 B}\right)\right.\right. \\
\left.+\int_{\min \left\{v_{0 A}+\theta, 1\right\}}^{1}\left(v_{0 B}-v_{1 B}\right) d F\left(v_{1 A} \mid v_{1 B}\right)\right] d F\left(v_{1 B}\right) \\
\left.+\int_{p}^{\max \left\{p, v_{0 A}\right\}}\left[\int_{v_{1 B}}^{v_{0 A}}\left(v_{0 A}-v_{1 A}\right) d F\left(v_{1 A} \mid v_{1 B}\right)\right] d F\left(v_{1 B}\right)\right] \\
+\operatorname{Pr}\left(v_{1 B} \geq v_{1 A}\right)\left[\int _ { p _ { c } } ^ { p } \left[\int_{v_{1 A}}^{\min \left\{v_{0 B}+\theta, 1\right\}}\left(v_{0 B}+\theta-v_{1 B}+v_{0 A}-v_{1 A}\right) d F\left(v_{1 B} \mid v_{1 A}\right)\right.\right. \\
\left.+\int_{p}^{1}\left(v_{\min \left\{v_{0 B}+\theta, 1\right\}} v_{0 A}-v_{1 A}\right) d F\left(v_{1 B} \mid v_{1 A}\right)\right] d F\left(v_{1 A}\right) \\
\max \left\{p, v_{0 A}\right\} \\
\left.\left.\int_{v_{1 A}}^{1}\left(v_{0 A}-v_{1 A}\right) d F\left(v_{1 B} \mid v_{1 A}\right)\right] d F\left(v_{1 A}\right)\right]
\end{array}
$$

The main difference from the main model is that the expected payoff function has two parts since the global bidder might win license $B$ first when $v_{1 A} \geq v_{1 B}$, and might win

\footnotetext{
${ }^{32}$ This makes the global bidder's license valuations heterogeneous.
} 
license $A$ first when $v_{1 B} \geq v_{1 A}$. Conditional on $v_{1 B} \geq v_{1 A}$, equation 12 describes when the global bidder wins both licenses, equation 13 describes when the global bidder wins license $A$ first but loses license $B$, and equation 14 describes when the global bidder drops out from license $B$ but wins license $A$ after. ${ }^{33}$ Note that equation 14 is not the symmetric equivalent of equation 11.

When $v_{0 A}+\theta<1$, the first derivative of this maximization problem is:

$$
\begin{array}{r}
F O C(p)=\operatorname{Pr}\left(v_{1 A} \geq v_{1 B}\right)\left[\int_{p}^{v_{0 A}+\theta}\left(V_{0}-p-v_{1 A}\right) f\left(v_{1 A} \mid p\right) d v_{1 A}\right. \\
\left.+\int_{v_{0 A}+\theta}^{1}\left(v_{0 B}-p\right) f\left(v_{1 A} \mid p\right) d v_{1 A}-\mathbb{1}_{\left[p<v_{0 A}\right]} \int_{p}^{v_{0 A}}\left(v_{0 A}-v_{1 A}\right) f\left(v_{1 A} \mid p\right) d v_{1 A}\right] f(p) \\
+\operatorname{Pr}\left(v_{1 B} \geq v_{1 A}\right)\left[\int_{p}^{v_{0 B}+\theta}\left(V_{0}-p-v_{1 B}\right) f\left(v_{1 B} \mid p\right) d v_{1 B}\right. \\
\left.+\int_{v_{0 B}+\theta}^{1}\left(v_{0 A}-p\right) f\left(v_{1 B} \mid p\right) d v_{1 B}-\mathbb{1}_{\left[p<v_{0 A}\right]}\left(v_{0 A}-p\right)\right] f(p)
\end{array}
$$

We can write $F O C(p)=J(p) f(p)$. By using the independence of $v_{1 A}$ and $v_{1 B}$, we calculate $\operatorname{Pr}\left(v_{1 A} \geq v_{1 B}\right)=0.5 .^{34}$

The first order condition is slightly different than the main model since equation 11 and 14 are not symmetric. ${ }^{35}$ Regardless, the optimal drop out price is unique. In the proofs, similar to the main model, we show that $J(p)$ is decreasing in $p$. Then, we will show that $J\left(p=v_{0 B}\right)>0$. The optimal drop out price $p$ will be less than or greater than $v_{0 A}$ depending on whether $J\left(p=v_{0 A}\right)<0$ or $J\left(p=v_{0 A}\right)>0$. The optimal drop out price $p_{0}^{*}$ is the solution to the first order condition of the global bidder's expected payoff maximization problem.

In Lemma 8 below, we characterize the global bidder's equilibrium bids. ${ }^{36}$

\footnotetext{
${ }^{33}$ Global bidder values $A$ more than $B$ so it will still drop out from license $B$ first. However, it might win license A first.

${ }^{34} \operatorname{Pr}\left(v_{1 A} \geq v_{1 B}\right)=\int_{0}^{1} \int_{0}^{v_{1 A}} f\left(v_{1 A}, v_{1 B}\right) d v_{1 B} d v_{1 A}=\int_{0}^{1} \int_{0}^{v_{1 A}} f\left(v_{1 A}\right) f\left(v_{1 B}\right) d v_{1 A}$

$=\int_{0}^{1}\left[\int_{0}^{v_{1 A}} f\left(v_{1 B}\right) d v_{1 B}\right] f\left(v_{1 A}\right) d v_{1 A}=\int_{0}^{1} F\left(v_{1 A}\right) f\left(v_{1 A}\right) d v_{1 A}$. Let $u=F\left(v_{1 A}\right)$, and $d u=f\left(v_{1 A}\right) d v_{1 A}$, we can write the last equation as $\int_{0}^{1} u d u=0.5$. Similarly, $\operatorname{Pr}\left(v_{1 B} \geq v_{1 A}\right)=0.5$.

${ }^{35}$ As a result, we do not have an intuitive Case I payoff since Case I payoff is based on the idea that the global bidder will win $B$ first. Here, it might win $A$ first.

${ }^{36}$ We could not generalize the result for cases in which there are more than one local bidder. If there are more than one local bidder, the global bidder has to update its optimal drop out price as some local
} 
Proposition 8 Suppose that the average valuation of the global bidder is less than 1 and there is one local bidder actively bidding on each license. The global bidder maximizes its payoff by dropping out of the license $B$ auction at the unique optimal drop-out price $p_{0}^{*}$ that solves $J(p)$ (and hence FOC), of the expected payoff function. Moreover,

a) If $v_{0 A}+\theta<1$, and $J\left(v_{0 A}\right)=\int_{v_{0 A}}^{v_{0 A}+\theta} F\left(v_{1 A} \mid v_{0 A}\right) d v_{1 A}+\left(v_{0 B}-v_{0 A}\right)+\int_{v_{0 A}}^{v_{0 B}+\theta} F\left(v_{1 B} \mid v_{0 A}\right) d v_{1 B}<$ 0 , then $p_{0}^{*}<v_{0 A}$. The global bidder will stay in the license $A$ auction until $v_{0 A}$ (after dropping out from the license $B$ auction).

b) If $v_{0 A}+\theta<1$ and $J\left(v_{0 A}\right)=\int_{v_{0 A}}^{v_{0 A}+\theta} F\left(v_{1 A} \mid v_{0 A}\right) d v_{1 A}+\left(v_{0 B}-v_{0 A}\right)+\int_{v_{0 A}}^{v_{0 B}+\theta} F\left(v_{1 B} \mid v_{0 A}\right) d v_{1 B} \geq$ 0 , then $p_{0}^{*} \geq v_{0 A}$ and the global bidder will also drop out of the license $A$ auction at $p_{0}^{*}$.

c) If $v_{0 A}+\theta \geq 1, v_{0 B}+\theta<1$, and $J\left(v_{0 A}\right)=\int_{v_{0 A}}^{1} F\left(v_{1 A} \mid v_{0 A}\right) d v_{1 A}+\left(v_{0 B}+\theta-1\right)+$ $\int_{v_{0 A}}^{v_{0 B}+\theta} F\left(v_{1 B} \mid v_{0 A}\right) d v_{1 B}<0$, then $p_{0}^{*}<v_{0 A}$. The global bidder will stay in the license $A$ auction until $v_{0 A}$ (after dropping out from the license $B$ auction).

d) Assume that $v_{0 A}+\theta \geq 1$,

i) If $v_{0 B}+\theta<1$, and $J\left(v_{0 A}\right)=\int_{v_{0 A}}^{1} F\left(v_{1 A} \mid v_{0 A}\right) d v_{1 A}+\left(v_{0 B}+\theta-1\right)+\int_{v_{0 A}}^{v_{0 B}+\theta} F\left(v_{1 B} \mid v_{0 A}\right) d v_{1 B} \geq$ 0 ,

$\mathrm{Or}$

ii) If $v_{0 B}+\theta \geq 1$,

then $v_{0 A} \leq p_{0}^{*} \leq 1$ and the global bidder will also drop out of the license $A$ auction at $p_{0}^{*}$.

We are ready to summarize our Bayesian-Nash equilibrium.

\section{Proposition 9 (Bayesian-Nash Equilibrium)}

a) A local bidder of each license will stay in the auction $j$ until the price reaches her valuation $v_{i j}$ where $j=\{A, B\}, i=\{1,2, \ldots m\}$.

b) A global bidder active only on license $j$ will bid $v_{0 j}+\theta$, if he won the other license. He will bid $v_{0 j}$ if he dropped out of the other license.

c) When the average valuation is less than one, the global bidder who is active on both licenses will drop out from license $B$ and/or $A$ as described in Proposition 8.

bidders drop out from the auction. We could not show that this updated price increases with the exit of local bidders. Given the possibility that the updated price might decrease makes this a complex problem. 


\begin{tabular}{cccccc} 
& License A won by & License B won by & Global bidder makes & Allocation is & Revenue SAA \\
\hline 1. & Global Bidder & Global Bidder & Profit & Efficient & $p_{A}+p_{B}$ \\
2. & Global Bidder & Global Bidder & Loss & Inefficient & $p_{A}+p_{B}$ \\
3. & Global Bidder & Local Bidder B & Profit & Efficient & $p_{A}+v_{0 B}+\theta$ \\
4. & Global Bidder & Local Bidder B & Loss & Inefficient & $p_{A}+v_{0 B}+\theta$ \\
5. & Global Bidder & Local Bidder B & Profit & Efficient & $p_{A}+p_{0}^{*}$ \\
6. & Global Bidder & Local Bidder B & Profit & Inefficient & $p_{A}+p_{0}^{*}$ \\
7. & Local Bidder A & Global Bidder & (not possible) & N/A & N/A \\
8. & Local Bidder A & Local Bidder B & Zero Profit & Efficient & $p_{0}^{*}+v_{0 A}$ \\
9. & Local Bidder A & Local Bidder B & Zero Profit & Inefficient & $p_{0}^{*}+v_{0 A}$ \\
10. & Local Bidder A & Local Bidder B & Zero Profit & Efficient & $2 p_{0}^{*}$ \\
11. & Local Bidder A & Local Bidder B & Zero Profit & Inefficient & $2 p_{0}^{*}$
\end{tabular}

Table 4: All efficient and inefficient outcomes for $v_{1 A}<v_{1 B}$ case.

d) If the average valuation is greater than one, the global bidder will stay in both auctions until price reaches his average valuation. ${ }^{37}$

We summarize all possible efficient and inefficient outcomes for $v_{1 A}<v_{1 B}$ case in Table 4. By using Table 1 and Table 4, we can count the efficient and inefficient outcomes in our simulation code. The results are summarized in figures 3 and 4 at the end of the paper. The Ex-Post Loss Probability are the probability of cases in which the global bidder makes a loss. The Probability of Inefficiency are the cases of all inefficient allocations including the ex-post loss probability cases.

One difference between the original model and this model is the optimal drop out prices (see Table 2 and 6 for a comparison). In the current model, local bidder $A$ 's valuation is drawn independently so it is expected to be lower compared to main model which assigns the maximum valuation to license $A$. This makes the global bidder use a slightly higher optimal drop out price, and decreases the probability of local bidders winning inefficiently. The ex-post loss probability of global bidder is also lower since global bidder is more likely to win license $A$ after winning license $B$, and at a lower price. As a result, it decreases the probability of inefficient allocations.

We also report the percentage decrease in total welfare with the SAA auction compared

\footnotetext{
${ }^{37}$ Price does not have to stop increasing at 1 . In the equilibrium outcome, the game will end before the price exceeds 1 .
} 
Table 5: Percentage Shortfall in Welfare in the SAA

Relative to the VCG Auction

\begin{tabular}{cccc}
\hline \hline Synergy & Uniform Distr. & $\begin{array}{c}\text { Beta Distr.with } \alpha=5 \\
\text { and } \beta=5\end{array}$ & $\begin{array}{c}\text { Beta Distr.with } \alpha=1 \\
\text { and } \beta=3\end{array}$ \\
\hline \hline 0.2 & $0.1002 \%$ & $0.1271 \%$ & $0.3025 \%$ \\
0.4 & $0.1925 \%$ & $0.1060 \%$ & $0.3860 \%$ \\
0.6 & $0.1577 \%$ & $0.0370 \%$ & $0.1898 \%$ \\
0.8 & $0.1045 \%$ & $0.0055 \%$ & $0.0616 \%$ \\
\hline \hline
\end{tabular}

to the VCG auction in Table $5 .^{38}$ The revised model is the model where any auction might end with a positive probability, and the main model is the model discussed in Section 4. The expected welfare loss is less than one per cent in all distributions we have used. While this may seem low, the revenue of the 2008 Canadian AWS auction was more than 4 billion dollars. Even a small percentage decrease (say 0.5 per cent) might have a relatively big magnitude effect (more than 20 million dollars).

Finally, we note that the results of Propositon 6 and 7 are valid for the current model. Lemma 5 has to be revised since the global bidder might win license $A$ first. In this case, if the global bidder has an ex-post loss, then $p_{0}^{*}>p_{A}=v_{1 A}>v_{0 A}$ or $p_{0}^{*}>p_{B}=v_{1 B}>v_{0 B}$ (depending on which license is won first) has to hold, and the outcome is inefficient. We do not provide the proofs since they are similar to the proof of Lemma 5, Proposition 6 and 7 .

\section{Proof of Proposition 8.}

Similar to Proposition 1, we will prove that there is a unique optimal drop-out price with a series of lemmas. First we will show that there exists a unique $p_{0}^{*} \in(0,1)$ that solves $J\left(p_{0}^{*}\right)=0$. Then, we will prove that only 0 and $p_{0}^{*}$ satisfy $F O C=J(p) f(p)=0$. Then, we will show that $p_{0}^{*}$ satisfies the second order condition so it is a local maximum. Then, we will show that 0 cannot be the maximizer, and that, $p_{0}^{*}$ is the unique maximizer.

\footnotetext{
${ }^{38}$ We thank a referee for asking us to calculate the welfare decrease.
} 
Table 6: GLOBAL BIDDER'S OPTIMAL DROP-OUT PRICE FOR THE REVISED MODEL FOR VARIOUS DISTRIBUTIONS AND SYNERGY LEVELS

\begin{tabular}{|c|c|c|c|c|}
\hline $\begin{array}{c}\text { Global } \\
\text { Bidder's } \\
\text { Valuation } \\
\text { for License A } \\
v_{0 A} \\
\end{array}$ & $\begin{array}{c}\text { Global } \\
\text { Bidder's } \\
\text { Valuation } \\
\text { for License B } \\
v_{0 B} \\
\end{array}$ & $\begin{array}{l}\text { Uniform } \\
\text { Distr. }\end{array}$ & $\begin{array}{c}\text { Beta } \\
\text { Distr. } \\
\text { with } \alpha=5 \\
\text { and } \beta=5 \\
p_{1}^{\beta=5}\end{array}$ & $\begin{array}{c}\text { Beta } \\
\text { Distr. } \\
\text { with } \alpha=1 \\
\text { and } \beta=3 \\
p_{1}^{\beta=3}\end{array}$ \\
\hline \multicolumn{5}{|c|}{ Synergy $=0.2$} \\
\hline 0.25 & 0.2 & 0.2441 & 0.2447 & 0.2690 \\
\hline 0.4 & 0.2 & 0.2734 & 0.2981 & 0.3262 \\
\hline 0.6 & 0.2 & 0.3172 & 0.3721 & 0.3765 \\
\hline 0.8 & 0.2 & 0.3691 & 0.3990 & 0.3982 \\
\hline 0.81 & 0.4 & 0.5607 & 0.5980 & 0.5952 \\
\hline \multicolumn{5}{|c|}{ Synergy $=0.4$} \\
\hline 0.25 & 0.2 & 0.3007 & 0.3317 & 0.3536 \\
\hline 0.4 & 0.2 & 0.3806 & 0.4332 & 0.4396 \\
\hline 0.6 & 0.2 & 0.4667 & 0.5472 & 0.5429 \\
\hline 0.8 & 0.2 & 0.5569 & 0.5973 & 0.5941 \\
\hline 0.81 & 0.4 & 0.7383 & 0.7826 & 0.7752 \\
\hline \multicolumn{5}{|c|}{ Synergy =0.6 } \\
\hline 0.25 & 0.2 & 0.3836 & 0.4542 & 0.4581 \\
\hline 0.4 & 0.2 & 0.4795 & 0.5478 & 0.5441 \\
\hline 0.6 & 0.2 & 0.6174 & 0.6668 & 0.6601 \\
\hline 0.8 & 0.2 & 0.7333 & 0.7791 & 0.7714 \\
\hline 0.81 & 0.4 & 0.8733 & 0.8958 & 0.8914 \\
\hline \multicolumn{5}{|c|}{ Synergy=0.8 } \\
\hline 0.25 & 0.2 & 0.5000 & 0.5773 & 0.5714 \\
\hline 0.4 & 0.2 & 0.6000 & 0.6653 & 0.6571 \\
\hline 0.6 & 0.2 & 0.7333 & 0.7791 & 0.7714 \\
\hline 0.8 & 0.2 & 0.8667 & 0.8903 & 0.8857 \\
\hline 0.81 & 0.4 & 1 & 1 & 1 \\
\hline
\end{tabular}


Figure 3: Beta Distribution $\alpha=1, \beta=3$.

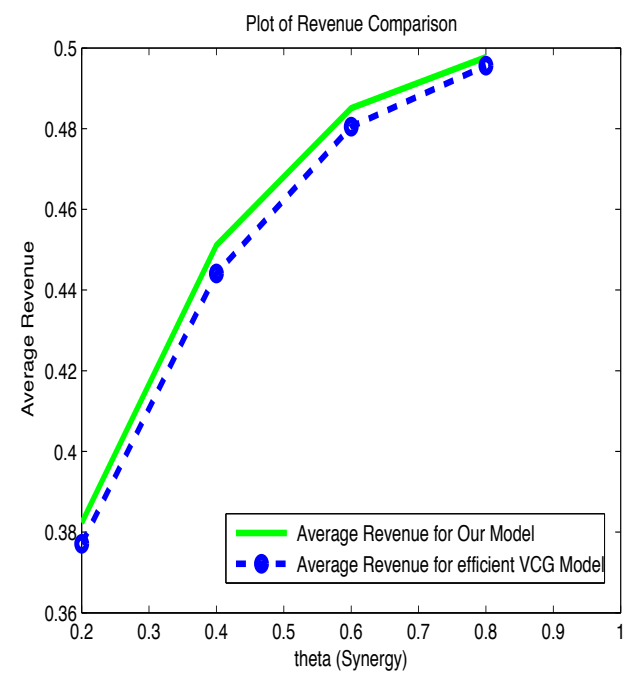

Lemma 10 There exists a unique $p_{0}^{*}$ such that $J\left(p_{0}^{*}\right)=0$ given the conditions a) to d) in Proposition 8.

Proof of Lemma 10 a). In this case, we have the assumptions of $v_{0 A}+\theta<1$ and $\left[\int_{v_{0 A}}^{v_{0 A}+\theta} F\left(v_{1 A} \mid v_{0 A}\right) d v_{1 A}+\left(v_{0 B}-v_{0 A}\right)+\int_{v_{0 A}}^{v_{0 B}+\theta} F\left(v_{1 B} \mid v_{0 A}\right) d v_{1 B}\right]<0$, we will show that this implies $v_{0 B}<p_{0}^{*}<v_{0 A}$.

We show that there exists a unique solution that solves $J(p)$. To prove uniqueness, we will show that this function is monotonically decreasing in $\left(0, v_{0 A}\right)$, and it is positive when $p=v_{0 B}$ and is negative when $p=v_{0 A}$. Hence, there must be a unique root in the interval $v_{0 B}<p<v_{0 A}$.

By taking the derivative of the expected payoff function, we get $J(p)=\operatorname{Pr}\left(p_{A} \geq\right.$ $\left.p_{B}\right)\left[\int_{p}^{v_{0 A}+\theta}\left(v_{0 A}+\theta-v_{1 A}+v_{0 B}-p\right) d F\left(v_{1 A} \mid p\right)+\int_{v_{0 A}+\theta}^{1}\left(v_{0 B}-p\right) d F\left(v_{1 A} \mid p\right)-\int_{p}^{v_{0 A}}\left(v_{0 A}-\right.\right.$ $\left.\left.v_{1 A}\right) d F\left(v_{1 A} \mid p\right)\right]+\operatorname{Pr}\left(v_{1 B} \geq v_{1 A}\right)\left[\int_{p}^{v_{0 B}+\theta}\left(v_{0 B}+\theta-v_{1 B}+v_{0 A}-p\right) d F\left(v_{1 B} \mid p\right)+\int_{v_{0 B}+\theta}^{1}\left(v_{0 A}-\right.\right.$ $\left.p) d F\left(v_{1 B} \mid p\right)-\int_{p}^{1}\left(v_{0 A}-p\right) d F\left(v_{1 B} \mid p\right)\right]$

Very similar to the proof of Proposition 1, we use integration by parts twice and use 
Figure 4: Top: Uniform Distribution. Bottom: Beta Distribution with $\alpha=\beta=5$
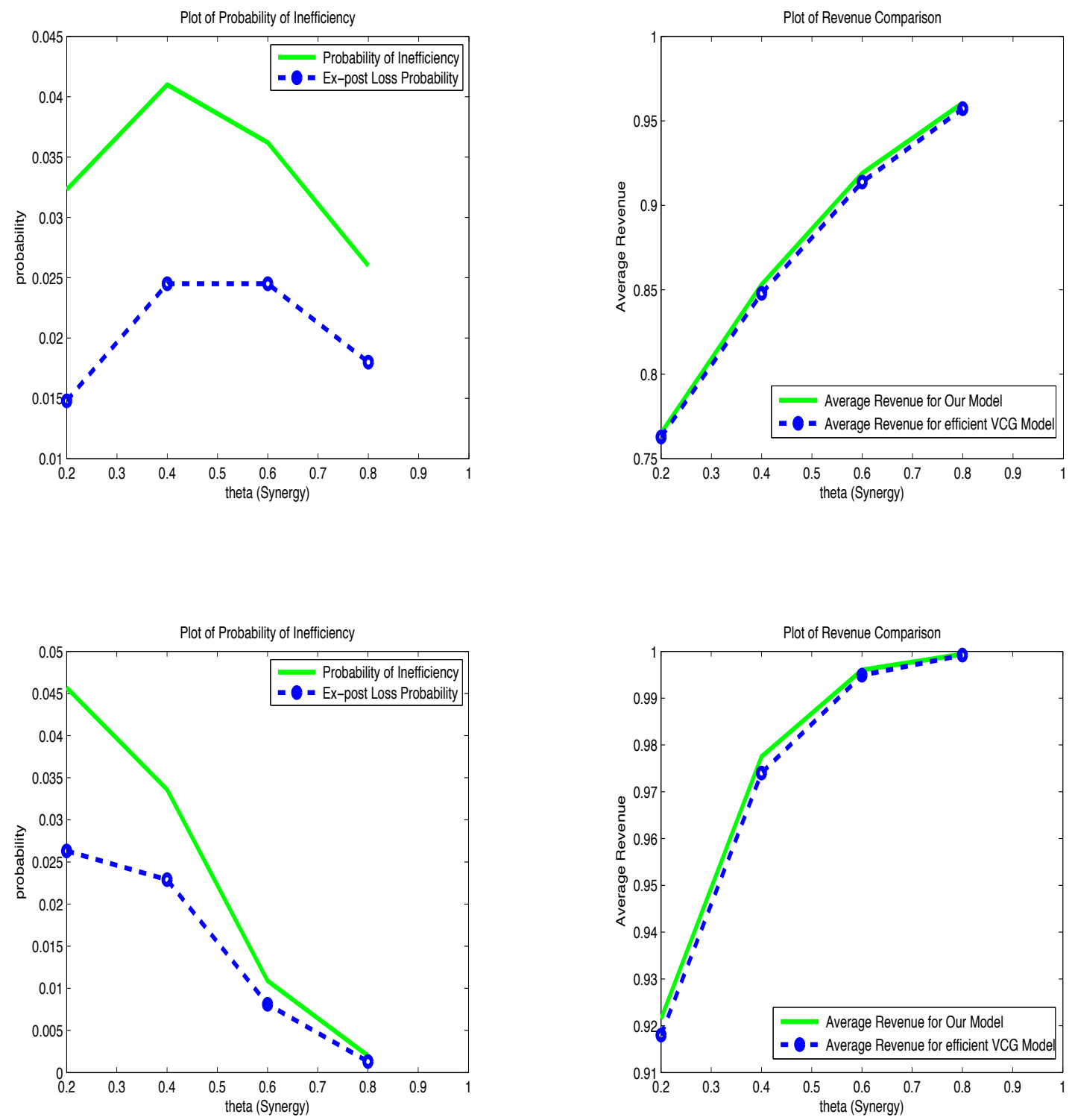
$\operatorname{Pr}\left(v_{1 A} \geq v_{1 B}\right)=0.5$, we can re-write

$$
\begin{gathered}
J(p)=0.5\left[-\int_{p}^{v_{0 A}}\left(v_{0 A}-p_{A}\right) f\left(v_{1 A} \mid p\right) d p_{A}+\int_{p}^{v_{0 A}+\theta}\left(v_{0 A}+\theta-v_{1 A}\right) f\left(v_{1 A} \mid p\right) d v_{1 A}+\left(v_{0 B}-p\right)+\right. \\
\left.\int_{p}^{v_{0 B}+\theta}\left(v_{0 B}+\theta-v_{1 B}\right) f\left(v_{1 B} \mid p\right) d v_{1 B}\right]=0.5\left[\int_{v_{0 A}}^{v_{0 A}+\theta} F\left(v_{1 A} \mid p\right) d v_{1 A}+\left(v_{0 B}-p\right)+\int_{p}^{v_{0 B}+\theta} F\left(v_{1 B} \mid p\right) d v_{1 B}\right]
\end{gathered}
$$

We take partial derivative of $J(p)$ with respect to $p$, we have,

$$
\frac{\partial J(p)}{\partial p}=0.5\left[\frac{\partial}{\partial p}\left[\int_{v_{0 A}}^{v_{0 A}+\theta} F\left(v_{1 A} \mid p\right) d v_{1 A}\right]-1+\frac{\partial}{\partial p}\left[\int_{p}^{v_{0 B}+\theta} F\left(v_{1 B} \mid p\right) d v_{1 B}\right]\right]<0 \text {. This is negative }
$$

since we have shown that a similar integral is negative in Proposition 1. While that integral has $G(. / p)$ in it, $F(. / p)$ is a special case of $G$ with one local bidder. Thus, $J(p)$ is a monotonically decreasing function of $p$ in $\left(0, v_{0 A}\right)$.

$$
\begin{aligned}
& \text { If } p=v_{0 B} \text {, then } J\left(p=v_{0 B}\right)=0.5\left[\int_{v_{0 A} A}^{v_{0 A}+\theta} F\left(v_{1 A} \mid v_{0 B}\right) d v_{1 A}+\int_{v_{0 B}}^{v_{0 B}+\theta} F\left(v_{1 B} \mid v_{0 B}\right) d v_{1 B}\right]>0 . \\
& \text { If } p=v_{0 A} \text {, then } J\left(p=v_{0 A}\right)=0.5\left[\int_{v_{0 A}+\theta}^{v_{0 A}} F\left(v_{1 A} \mid v_{0 A}\right) d v_{1 A}+\left(v_{0 B}-v_{0 A}\right)+\int_{v_{0 A}}^{v_{0 B}+\theta} F\left(v_{1 B} \mid v_{0 A}\right) d v_{1 B}\right]<
\end{aligned}
$$
0 by our assumption that $\int_{v_{0 A}}^{v_{0 A}+\theta} F\left(v_{1 A} \mid v_{0 A}\right) d v_{1 A}+\left(v_{0 B}-v_{0 A}\right)+\int_{v_{0 A}}^{v_{0 B}+\theta} F\left(v_{1 B} \mid v_{0 A}\right) d v_{1 B}<0$.

Hence, there is a unique root, $p_{0}^{*}$ in the such that $v_{0 B}<p_{0}^{*}<v_{0 A}$. When the global bidder drops out from license $B$ auction, it is straightforward to see that it is profitable for him/her to continue on license $A$ until price reaches $v_{0 A}$.

b) In this case, we have the assumptions of $v_{0 A}+\theta<1$ and $\left[\int_{v_{0 A}}^{v_{0 A}+\theta} F\left(v_{1 A} \mid v_{0 A}\right) d v_{1 A}+\right.$ $\left.\left(v_{0 B}-v_{0 A}\right)+\int_{v_{0 A}+\theta}^{v_{0 B}+\theta} F\left(v_{1 B} \mid v_{0 A}\right) d v_{1 B}\right] \geq 0$, we will show that this implies $v_{0 A} \leq p_{0}^{*}<v_{0 B}+\theta$. $J(p)=0.5\left[\int_{p}^{v_{0 A}+\theta}\left(v_{0 A}+\theta-v_{1 A}+v_{0 B}-p\right) d F\left(v_{1 A} \mid p\right)+\int_{v_{0 A}+\theta}^{1}\left(v_{0 B}-p\right) d F\left(v_{1 A} \mid p\right)\right]+$ $0.5\left[\int_{p}^{v_{0 B}+\theta}\left(v_{0 B}+\theta-v_{1 B}+v_{0 A}-p\right) d F\left(v_{1 B} \mid p\right)+\int_{v_{0 B}+\theta}^{1}\left(v_{0 A}-p\right) d F\left(v_{1 B} \mid p\right)\right]=0.5\left[\int_{p}^{v_{0 A}+\theta} F\left(v_{1 A} \mid p\right) d v_{1 A}+\right.$ $\left.\left(v_{0 B}-p\right)+\int_{p}^{v_{0 B}+\theta} F\left(v_{1 B} \mid p\right) d v_{1 B}\right]$. It can be shown that $J$ is monotonically decreasing in $p \in\left(v_{0 A}, 1\right)$

$$
\text { If } p=v_{0 A}, J\left(p=v_{0 A}\right)=0.5\left[\int_{v_{0 A}}^{v_{0 A}+\theta} F\left(v_{1 A} \mid v_{0 A}\right) d v_{1 A}+\left(v_{0 B}-v_{0 A}\right)+\int_{v_{0 A}}^{v_{0 B}+\theta} F\left(v_{1 B} \mid v_{0 A}\right) d v_{1 B}\right] \geq
$$
0 by our assumption that $\left[\int_{v_{0 A} A}^{v_{0 A}+\theta} F\left(v_{1 A} \mid v_{0 A}\right) d v_{1 A}+\left(v_{0 B}-v_{0 A}\right)+\int_{v_{0 A}}^{v_{0 B}+\theta} F\left(v_{1 B} \mid v_{0 A}\right) d v_{1 B}\right] \geq 0$.

If $p=v_{0 B}+\theta$, then $J\left(p=v_{0 B}+\theta\right)=0.5\left[\int_{v_{0 B}+\theta}^{v_{0 A}+\theta} F\left(v_{1 A} \mid v_{0 A}\right) d v_{1 A}-\theta<\int_{v_{0 B}+\theta}^{v_{0 A}+\theta} 1 d v_{1 A}-\theta\right]=$ $v_{0 A}-v_{0 B}-\theta \leq 0$.

Hence, there is a unique root, $p_{0}^{*}$ in the such that $v_{0 A} \leq p_{1}^{*}<v_{0 B}+\theta$.

c) In this case, we have the assumptions of $v_{0 A}+\theta \geq 1, v_{0 B}+\theta<1$, and $\left[\int_{v_{0 A}}^{1} F\left(v_{1 A} \mid v_{0 A}\right) d v_{1 A}+\right.$ $\left.\left(v_{0 B}+\theta-1\right)+\int_{v_{0 A}}^{v_{0 B}+\theta} F\left(v_{1 B} \mid v_{0 A}\right) d v_{1 B}\right]<0$, We will show that this implies $v_{0 B}<p_{0}^{*}<v_{0 A}$.

With some calculation similar to the proof of Proposition 1, we can show that $J(p)=$ 
$0.5\left[\int_{p}^{1}\left(v_{0 A}+\theta-v_{1 A}+v_{0 B}-p\right) d F\left(v_{1 A} \mid p\right)-\int_{p}^{v_{0 A}}\left(v_{0 A}-v_{1 A}\right) d F\left(v_{1 A} \mid p\right)\right]+0.5\left[\int_{p}^{v_{0 B}+\theta}\left(v_{0 B}+\theta-\right.\right.$ $\left.\left.v_{1 B}+v_{0 A}-p\right) d F\left(v_{1 B} \mid p\right)+\int_{v_{0 B}+\theta}^{1}\left(v_{0 A}-p\right) d F\left(v_{1 B} \mid p\right)-\int_{p}^{1}\left(v_{0 A}-p\right) d F\left(v_{1 B} \mid p\right)\right]=0.5\left[\int_{v_{0 A}}^{1} F\left(v_{1 A} \mid p\right) d v_{1 A}+\right.$ $\left.\left(v_{0 A}+v_{0 B}+\theta-p-1\right)+\int_{p}^{v_{0 B}+\theta} F\left(v_{1 B} \mid p\right) d v_{1 B}\right]$ and that $J(p)$ is decreasing in $\left(0, v_{0 A}\right)$.

If $p=v_{0 B}$, then $J\left(p=v_{0 B}\right)=0.5\left[\int_{v_{0 A}}^{1} F\left(v_{1 A} \mid v_{0 B}\right) d v_{1 A}+v_{0 A}+\theta-1+\int_{v_{0 B}}^{v_{0 B}+\theta} F\left(v_{1 B} \mid v_{0 B}\right) d v_{1 B}\right]>$ 0 , since $v_{0 A}+\theta \geq 1$ and $F() \leq$.1 .

If $p=v_{0 A}, J\left(p=v_{0 A}\right)=0.5\left[\int_{v_{0 A}}^{1} F\left(v_{1 A} \mid v_{0 A}\right) d v_{1 A}+\left(v_{0 B}+\theta-1\right)+\int_{v_{0 A}}^{v_{0 B}+\theta} F\left(v_{1 B} \mid v_{0 A}\right) d v_{1 B}\right]<$ 0 by our assumption that $\int_{v_{0 A}}^{1} F\left(v_{1 A} \mid v_{0 A}\right) d v_{1 A}+\left(v_{0 B}+\theta-1\right)+\int_{v_{0 A}}^{v_{0 B}+\theta} F\left(v_{1 B} \mid v_{0 A}\right) d v_{1 B}<0$.

Hence, there is a unique root, $p_{0}^{*}$ in the such that $v_{0 B}<p_{1}^{*}<v_{0 A}$.

d) i) In this case, $J(p)=0.5 \int_{p}^{1}\left(v_{0 A}+\theta-v_{1 A}+v_{0 B}-p\right) d F\left(v_{1 A} \mid p\right)+0.5\left[\int_{p}^{v_{0 B}+\theta}\left(v_{0 B}+\right.\right.$ $\left.\left.\theta-v_{1 B}+v_{0 A}-p\right) d F\left(v_{1 B} \mid p\right)+\int_{v_{0 B}+\theta}^{1}\left(v_{0 A}-p\right) d F\left(v_{1 B} \mid p\right)\right]=0.5\left[\int_{p}^{1} F\left(v_{1 A} \mid p\right) d v_{1 A}+\left(v_{0 A}+\right.\right.$ $\left.\left.v_{0 B}+\theta-p-1\right)+\int_{p}^{v_{0 B}+\theta} F\left(v_{1 B} \mid p\right) d v_{1 B}+\left(v_{0 A}-p\right)\right]$. Similar to part b), we can easily show that $J(p)$ is decreasing in $\left(v_{0 A}, 1\right)$.

If $p=v_{0 A}, J\left(p=v_{0 A}\right)=0.5\left[\int_{v_{0 A}}^{1} F\left(v_{1 A} \mid v_{0 A}\right) d v_{1 A}+\left(v_{0 B}+\theta-1\right)+\int_{v_{0 A}}^{v_{0 B}+\theta} F\left(v_{1 B} \mid v_{0 A}\right) d v_{1 B}\right] \geq$ 0 by our assumption that $\int_{v_{0 A}}^{1} F\left(v_{1 A} \mid v_{0 A}\right) d v_{1 A}+\left(v_{0 B}+\theta-1\right)+\int_{v_{0 A}}^{v_{0 B}+\theta} F\left(v_{1 B} \mid v_{0 A}\right) d v_{1 B} \geq 0$. If $p=1$, then $J(p=1)=\left(v_{0 A}+v_{0 B}+\theta-2\right)+v_{0 A}-1<0$ since the average valuation is less than one by our assumption.

Hence, there is a unique root, $p_{0}^{*}$ in the such that $v_{0 A} \leq p_{1}^{*}<1$.

ii) In this case, $J(p)=0.5\left[\int_{p}^{1}\left(v_{0 A}+\theta-v_{1 A}+v_{0 B}-p\right) d F\left(v_{1 A} \mid p\right)\right]+0.5\left[\int_{p}^{1}\left(v_{0 B}+\theta-v_{1 B}+\right.\right.$ $\left.\left.v_{0 A}-p\right) d F\left(v_{1 B} \mid p\right)\right]=0.5\left[\int_{p}^{1} F\left(v_{1 A} \mid p\right) d v_{1 A}+2\left(v_{0 A}+v_{0 B}+\theta-p-1\right)+\int_{p}^{1} F\left(v_{1 B} \mid p\right) d v_{1 B}\right]$. Similar to part a), the first order condition is decreasing.

$$
\text { If } p=v_{0 A}, J\left(p=v_{0 A}\right)=0.5\left[\int_{v_{0 A}}^{1} F\left(v_{1 A} \mid v_{0 A}\right) d v_{1 A}+2\left(v_{0 B}+\theta-1\right)+\int_{v_{0 A}}^{1} F\left(v_{1 B} \mid v_{0 A}\right) d v_{1 B}\right] \geq
$$
0 by our assumption that $v_{0 B}+\theta \geq 1$. If $p=1$, then $J(p=1)=\left(v_{0 A}+v_{0 B}+\theta-2\right)<0$ by our assumption that the average valuation is less than 1.

Hence, there is a unique root, $p_{0}^{*}$ in the such that $v_{0 A} \leq p_{0}^{*}<1$. In this case $p_{0}^{*}$ cannot be less than $v_{0 A}$, since $\left[\int_{v_{0 A}}^{1} F\left(v_{1 A} \mid v_{0 A}\right) d v_{1 A}+2\left(v_{0 B}+\theta-1\right)+\int_{v_{0 A}}^{1} F\left(v_{1 B} \mid v_{0 A}\right) d v_{1 B}\right]$ is always positive.

Lemma 11 a) Only 0 and $p_{0}^{*}$ solves the FOC. 
b) $p_{0}^{*}$ satisfies the second order condition.

c) 0 cannot maximize the expected payoff.

Proof a) From lemma 10, we can write the $F O C=J(p) f(p)$ with the abuse of notation since $J(p)$ takes different forms in part a to d. It is easy to see that FOC is equal to zero only if $J(p)=0$ or $f(p)=0 .{ }^{39}$ In lemma 10 , we showed that there is a unique $p_{0}^{*}$ that makes $J(p)=0$. Hence, $F O C\left(p_{0}^{*}\right)=0$. This proves $p_{0}^{*}$ solves FOC.

By our assumption $f(p)=0$ only if $p=0$. Hence, only 0 , and $p_{0}^{*}$ solves the FOC.

b) $F O C=J(p) f(p)$, hence $S O C=J^{\prime}(p) f(p)+J(p) f^{\prime}(p)$. When $p=p_{0}^{*}, J\left(p_{0}^{*}\right)=0$ as shown in lemma 10, hence, $S O C=J^{\prime}\left(p_{0}^{*}\right) f\left(p_{0}^{*}\right)$. This is negative since we have showed in lemma 10 that $J(p)$ is a decreasing function and $f\left(p_{0}^{*}\right)>0$ for any $p \in(0,1)$. Note that $p_{0}^{*} \in(0,1)$.

c) It is straightforward to see that it cannot be optimal to drop out from license $B$ at $p=0$. The proof is similar to the one in lemma 11 part c so we skip it.

\footnotetext{
${ }^{39}$ One difference from the proof of Proposition 1 is that $m_{A}=1$ in this model; hence, 1 does not solve $h($. and hence, $F O C$.
} 